\title{
二核铌钼硫簇 $\mathrm{NbMoS}_{n}^{-10}(n=3 \sim 7)$ 掺杂体系的 结构与成键性质的理论研究
}

\author{
王涁*,a 王剑福 ${ }^{a} \quad$ 张晓菲 $^{a}$ 陈文杰 ${ }^{b}$ 章永凡 ${ }^{a}$ 黄昕 $^{a}$ \\ ( ${ }^{a}$ 福州大学化学学院 福州 350116) \\ ( ${ }^{b}$ 泉州师范学院化工与材料学院 泉州 362000)
}

\begin{abstract}
摘要 近年来, 过渡金属硫化物作为催化材料在许多化学反应中扮演着重要角色, 特别是在石油化工领域的加氢脱硫 与加氢脱氮等环节中被广泛应用. 本工作采用密度泛函理论结合高精度的耦合簇 $[C C S D(T)]$ 计算方法, 对掺杂类型的 铌钼硫簇 $\mathrm{NbMoS}_{n}^{-/ 0}(n=3 \sim 7)$ 进行系统研究, 确定其最稳定的几何结构, 并探讨掺杂、调节硫含量、改变团簇所带电 荷等手段, 对掺杂类型铌钼硫簇几何构型、电子结构与化学成键等性质的影响. 本工作采用广义 Koopmans 定理计算 $\mathrm{NbMoS}_{n}^{-}(n=3 \sim 7)$ 阴离子基态的电子垂直逸出能(VDEs), 模拟相应的阴离子光电子能谱图(PES), 并结合对分子轨道 的分析来进一步阐述该体系在几何结构与化学成键等性质上的演变规律. 本工作可为进一步开展铌钼硫簇掺杂体系的 理论与实验研究提供较为可靠的理论依据.
\end{abstract}

关键词 过渡金属硫簇; 密度泛函理论; 结构演化; 光电子能谱模拟

\section{Theoretical Investigations on the Structures and the Chemical Bonding of $\mathrm{NbMoS}_{n}^{-10}(n=3 \sim 7)$ Clusters}

\author{
Wang, Bin*,a Wang, Jianfu ${ }^{a} \quad$ Zhang, Xiaofei $^{a} \quad$ Chen, Wenjie $^{b}$ \\ Zhang, Yongfan ${ }^{a}$ Huang, $\mathrm{Xin}^{a}$ \\ ( ${ }^{a}$ College of Chemistry, Fuzhou University, Fuzhou 350116) \\ ( ${ }^{b}$ College of Chemical Engineering and Material, Quanzhou Normal University, Quanzhou 362000)
}

\begin{abstract}
Recently, transition metal sulfides (TMS) have played an important role in many catalytic reactions. In particular, they are widely used in the petrochemical industry, such as the hydrodesulfurization (HDS) and the hydrodenitrogenation (HDN) processes. In this work, density functional theory (DFT) and coupled cluster theory $[\mathrm{CCSD}(\mathrm{T})]$ calculations were used to study the niobium-mixed di-nuclear molybdenum sulfide clusters $\operatorname{NbMoS}_{n}^{-/ 0}(n=3 \sim 7)$. In our calculations, their ground-state structures were determined and the effects of doping metal, adjusting the sulfur content $(n)$ and changing the charge states of clusters were discussed on the geometries, electronic structures and chemical bonding of $\operatorname{NbMoS}_{n}^{-/ 0}(n=$ $3 \sim 7) . \operatorname{NbMoS}_{n}^{-/ 0}(n=3 \sim 7)$ clusters can be viewed as linking different sulfur ligands to the $\mathrm{NbMoS}_{2}$ four-membered rings. Among them, diverse poly-sulfur ligands, such as bridging $S_{2}$, terminal $S_{2}$ and terminal $S_{3}$ groups, emerged in the sulfur-rich clusters. Generalized Koopmans' Theorem was employed to predict the vertical detachment energies (VDEs), and simulate the corresponding anionic photoelectron spectra (PES). The first VDEs $\left(\operatorname{VDE}_{1 \mathrm{st}}\right)$ of $\operatorname{NbMoS}_{n}^{-}(n=3 \sim 6)$ increased gradually as a function of $n$, and then decreased suddenly when the sulfur content $(n)$ reached 7 . The $\operatorname{VDE}_{1 \mathrm{st}}$ reached the maximum by $4.69 \mathrm{eV}$ when the sulfur content equaled to 6 . The driving forces $(-\Delta G)$ of the reduction reactions between $\operatorname{NbMoS}_{n}^{-/ 0}(n$ $=3 \sim 7$ ) and $\mathrm{H}_{2}$ were evaluated. The $\mathrm{NbMoS}_{7}^{-}$anion with the terminal $\mathrm{S}_{2}^{2-}$ group yielded the negative value of $\Delta G$, which indicated that the reaction is thermodynamically favored even at the room temperature. We predicted that doping niobium into the molybdenum sulfides may improve the emergence of $\mathrm{S}_{2}$ group which may be helpful in producing the coordinatively unsaturated sites (CUS) under the $\mathrm{H}_{2} / \mathrm{H}_{2} \mathrm{~S}$ atmosphere. Molecular orbital analyses are performed to improve our understanding on the structural evolution and the chemical bonding of $\operatorname{NbMoS}_{n}^{-/ 0} \quad(n=3 \sim 7)$ clusters.

Keywords transition metal sulfide cluster; density functional theory; structural evolution; simulation of photoelectron spectrum
\end{abstract}

\footnotetext{
*E-mail: wangbin_100@fzu.edu.cn; Tel: 0086-0591-22866154

Received November 1, 2016; published December 20, 2016.

Supporting information for this article is available free of charge via the Internet at http://sioc-journal.cn.

Project supported by the National Natural Science Foundation of China (Nos. 21301030, 21371034, 21373048 and 21603117) and the Natural Science Foundation of Fuzhou University (2012-XY-6).

项目受国家自然科学基金(Nos. 21301030, 21371034, 21373048 和 21603117)与福州大学自然科学基金(2012-XY-6)资助.
} 


\section{1 引言}

近年来, 过渡金属硫化物(TMS)广泛应用于工业生 产与生物科学等领域 ${ }^{[1-5]}$, 受到人们越来越多的关注. 例如 VIB 族的钼硫化物在固体润滑剂、储氢材料以及工 业催化等方面有着重要应用 ${ }^{[6 \sim 13]}$. 其中, 以 $\mathrm{MoS}_{2}$ 作为 主催化成分的催化剂已实现在加氢脱硫(HDS)等石油炼 制过程中的商业化应用 ${ }^{[14,15]}$. 此外, 钼硫化物的价格相 对低廉、储量丰富, 可以代替贵金属(如铂族贵金属), 应 用于电催化析氢反应(HER)等过程中 ${ }^{[16 ~ 19]}$. 相比 VIB 族 的钼硫化物, 与其相邻的 VB 族铌硫化物在超导 ${ }^{[20,21]}$ 、 离子电池 ${ }^{[22]}$ 、湿度传感器 ${ }^{[23]}$ 、催化材料 ${ }^{[24]}$ 等方面也有着 大量的研究. 在 1-丁烯的异构化和氢化反应中, $\mathrm{NbS}_{2}$ 起 到了很好的催化效果 ${ }^{[25]}$. 而且作为一种非常有应用前 景的氢处理(HDT)催化材料, 它在很多反应中甚至表现 出了比传统的钼硫化物更高的催化活性 ${ }^{[26]}$. 有研究表 明在氢处理(HDT)反应中它们的催化活性表现出如下强 弱顺序: $\mathrm{NbS}_{3}>\mathrm{NbS}_{2}>\mathrm{Nb}_{1+} \mathrm{S}_{2}>\mathrm{MoS}_{2}{ }^{[27 \sim 31]}$. 虽然铌硫 化物表现出比钼硫化物更高的催化活性, 但由于铌硫化 物的天然矿物储量少, 合成时前驱体硫化困难, 且铌硫 化物在空气中容易被氧化 ${ }^{[32]}$, 因而大大限制了铌硫化 物的商业化应用与研究. 不过, 有研究表明将铌硫化物 掺杂到传统的 NiMo 硫化物催化剂中可显著提高其催化 活性 ${ }^{[33]}$. 这也说明了同族金属间掺杂或毗邻族金属间 掺杂有可能在很大程度上影响、改变化合物的几何构型 和电子结构, 进而影响其物理化学性质.

一般而言, 催化剂的性能与催化剂表面的活性位状 况有着密切的关系 ${ }^{[34]}$. 在这类过渡金属硫化物催化材 料的活性位研究方面, 前人做了许多工作. Topsøe 等 ${ }^{[35]}$ 提出 $\mathrm{MoS}_{2}$ 表面的边缘位置对 HDS 催化过程有着不可忽 视的作用. 特别是有不少研究指出 HDS 活性中心与催 化剂表面的配位不饱和位(CUS)密切相关, 但也有研究 指出在不含配位不饱和位(CUS)的催化剂表面也具有相 应的催化活性 ${ }^{[30,36 ~ 40]}$. 另有研究表明铌硫化物在 HDT 反应中所表现的高活性与其表面的 $\mathrm{S}_{2}^{2-}$ 基团以及 $\mathrm{Nb}^{\mathrm{IV}}-\mathrm{Nb}^{\mathrm{IV}}$ 阳离子对有关 ${ }^{[26]}$. 尽管前人已对催化活性位 的研究取得了不少进展, 但目前人们依然对活性位的精 细结构等存在着争议 ${ }^{[16,28]}$.

气相团簇作为连接宏观与微观世界的桥梁, 常被用 作研究块材体系的理论分子模型. 早在 20 世纪末, 黄建 全、卢嘉锡等 ${ }^{[41]}$ 科学家就提出了 $\left[\mathrm{Mo}_{3} \mathrm{~S}_{4}\right]^{4+}$ 簇骼中 $\mathrm{Mo}_{3} \mathrm{~S}_{3}$ 簇环具有类芳香性本质. 随后厦门大学的黄荣涁等 ${ }^{[42]}$ 通过质谱分析实验认为 $\left[\mathrm{Nb}_{3} \mathrm{~S}_{4}\right]^{+}$簇合物也具有类芳香性 的 $\mathrm{Nb}_{3} \mathrm{~S}_{3}$ 簇环. 值得注意的是, 由于铌 $\left(4 \mathrm{~d}^{4} 5 \mathrm{~s}^{1}\right)$ 与钿 $\left(4 d^{5} 5 s^{1}\right)$ 是同一周期互为邻族的过渡金属元素, 那么 $\left[\mathrm{Nb}_{3} \mathrm{~S}_{4}\right]^{+}$与 $\left[\mathrm{Mo}_{3} \mathrm{~S}_{4}\right]^{4+}$ 就具有相同的价电子数目. 此外, Gemming 等 ${ }^{[43 \sim 46]}$ 借助实验光电子能谱(PES)和理论计算 模拟相结合的手段, 对一系列一核到多核钼硫簇的结构 和性质进行了研究, 提供了部分钼硫簇的实验光电子能
谱数据. 中科院山西煤炭化学研究所的焦海军研究员与 其合作者们 ${ }^{[47]}$ 指出 $\mathrm{Mo}_{3} \mathrm{~S}_{9}$ 簇具有较小的 HOMO-LUMO 能隙值, 既可以作为 Lewis 酸也可以作为 Lewis 碱, 并 提出该团簇可以作为研究无定形 $\mathrm{MoS}_{3}$ 材料的理论模型. 此外, Bertram 等 ${ }^{[8]}$ 还利用紫外光电子能谱实验结合密 度泛函理论计算方法对幻数团簇 $\mathrm{M}_{4} \mathrm{X}_{6}(\mathrm{M}=\mathrm{W}, \mathrm{Mo}$, $\mathrm{X}=\mathrm{O}, \mathrm{S})$ 进行研究, 确定了 $\mathrm{Mo}_{4} \mathrm{~S}_{6}$ 的结构, 指出其具有 较大的 HOMO-LUMO 能隙值. Andrews 小组 ${ }^{[49,50]}$ 结合红 外光谱实验与密度泛函理论计算, 研究了一系列单核 $\mathrm{MS}_{n}(\mathrm{M}=\mathrm{V}, \mathrm{Nb}, \mathrm{Ta}, n=1 \sim 2 ; \mathrm{M}=\mathrm{Cr}, \mathrm{Mo}, \mathrm{W}, n=1 \sim 3)$ 团簇体系, 计算了其 $\mathrm{M}-\mathrm{S}$ 键的特征振动频率. 目前, 人们对铌硫簇的研究还比较有限, 研究多集中在与铌同 族的钒的硫化物上 ${ }^{[51,52]}$. 对于掺杂铌钼硫体系, 近年来 Saha 与 Raghavachari ${ }^{[53,54]}$ 报道了二元过渡金属硫族 $\mathrm{M}_{2} \mathrm{~S}_{x}^{-}(\mathrm{M}=\mathrm{Mo}, \mathrm{W}, x=4 \sim 6)$ 以及三元过渡金属硫簇 $\mathrm{MoWS}_{4}{ }^{-}$掺杂体系的稳定构型, 并对它们与水分子反应 的反应机理进行了研究. Afanasiev 等 ${ }^{[55]}$ 合成了 $\mathrm{Nb}_{2} \mathrm{Mo}_{3} \mathrm{~S}_{10}$ 并对其催化性质进行了表征，指出该物质在 噻吩加氢脱硫反应中的催化活性位于 $\mathrm{MoS}_{2}$ 和 $\mathrm{NbS}_{2}$ 之 间, 而且比它们都更加稳定. Gaborit 等 ${ }^{[56]}$ 对不同铌钿掺 杂比例的 $\mathrm{Nb}_{x} \mathrm{Mo}_{1-x} \mathrm{~S}_{2}$ 催化活性进行了比较, 最终认为当 $x=0.4$ 时其催化活性最高, 且掺 $\mathrm{Nb}$ 后其催化活性受 $\mathrm{H}_{2} \mathrm{~S}$ 的抑制作用会降低. 另外 Aray 等 ${ }^{[57]}$ 的研究也表明 $\mathrm{MoS}_{2}$ 催化剂表面掺 $\mathrm{Nb}$ 有助于提高其热力学稳定性. Ivanovskaya 等 ${ }^{[58,59]}$ 对掺杂 $\mathrm{Nb}$ 后 $\mathrm{MoS}_{2}$ 纳米管的稳定性、 结构与电子性质进行了研究, 表明掺杂 $\mathrm{Nb}$ 不仅有利于 提高 $\mathrm{MoS}_{2}$ 纳米管的稳定性, 而且掺杂后使纳米管具有 金属特性. Deepak 等 ${ }^{[60]}$ 实验合成了类富勒烯纳米颗粒 $\mathrm{Nb}_{x} \mathrm{Mo}_{1-x} \mathrm{~S}_{2}$, 并对其进行实验表征, 发现其在低温下具 有单电子隧道效应. 尽管目前人们对铌钿硫体系的研究 做了大量工作, 但掺杂体系的理论研究仍比较缺乏, 因 此, 我们希望通过对铌钿硫团簇 $\mathrm{NbMoS}_{n}^{-/ 0}(n=3 \sim 7)$ 掺 杂体系的研究, 分析掺杂、调节硫含量、改变团簇所带 电荷等手段对团簇几何构型、电子结构、化学成键等性 质的影响, 探索其特征规律与催化活性间的联系, 帮助 我们从分子层面加深对这类铌钼硫掺杂体系的了解, 有 助于我们进一步解释发生在这类材料表面的各种理化 过程, 例如加深对其在加氢脱硫等催化过程中构效关系 的理解, 为该类功能材料的性能改进提供较为可靠的理 论依据.

\section{2 计算方法}

本论文的计算工作使用 Gaussian 03 软件 ${ }^{[61]}$. 采用 B3LYP 杂化密度泛函 ${ }^{[62 ~ 64]}$, 先对 $\operatorname{NbMoS}_{n}^{-/ 0}(n=3 \sim 7)$ 体系的所有原子 $(\mathrm{Nb}, \mathrm{Mo}$ 和 $\mathrm{S}$ )使用 def2-TZVP 赝势及基 组(记作 B3LYP/Basis-I $)^{[65 \sim 67]}$, 搜索体系势能面, 确定最 稳定构型及与其能量相近的异构体. 然后, 对上述计算 得到的相对能量在 $0.50 \mathrm{eV}$ 以内的结构采用更高水平的 
赝势及基组进行优化, 即对 $\mathrm{Nb}$ 和 $\mathrm{Mo}$ 原子使用考虑了 标量相对论效应的 Stuttgart 有效核势(ECP)及基组 ${ }^{[68,69]}$, 并在该基组中添加了两个 $f$ 型和一个 $g$ 型的极化函数 $\mathrm{Nb} /[\zeta(f)=0.261,0.970 ; \zeta(g)=0.536], \mathrm{Mo} /[\zeta(f)=0.338$,

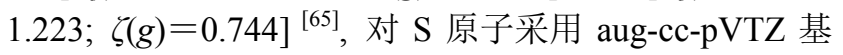

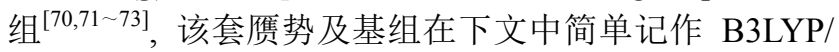
Basis-II. 对于其中能量极其靠近, 与最稳定构型可能存 在竞争关系的异构体, 我们采用更高计算精度的耦合族 [CCSD(T) $]^{\text {方法 }}{ }^{[74 ~ 78]}$ (利用 Molpro 2010.1 软 件 ${ }^{[79]}$ )来进 行检验. 最终确定了较为可靠的基态结构. 计算中各驻 点(stationary point)的性质是通过计算振动频率来确认. 体系各构型的三维分子轨道图由 Gauss View 4.1 软件 ${ }^{[80]}$ 绘制完成.

由于光电子能谱 (PES) 可作为辨别团簇, 获得团簇 电子结构信息的有效工具, 所以在确定 $\mathrm{NbMoS}_{n}^{-/ 0}(n=$ 3 7)体系基态构型的基础上, 我们模拟了各阴离子的 光电子能谱图, 以期为将来 $\operatorname{NbMoS}_{n}^{-}(n=3 \sim 7)$ 体系光 电子谱图的辨析与进一步解释提供数据支持. 在模拟光 电子能谱过程中所需的垂直电子逸出能(VDE)的计算, 是基于广义 Koopmans 定理(GKT), 即在阴离子轨道能 量本征值基础上加上一个校正项 $\delta E=E_{1}-E_{2}-\varepsilon_{\mathrm{HOMO}}{ }^{[81]}$, 其中 $E_{1}$ 和 $E_{2}$ 分别代表阴离子基态及其中性团簇在阴离 子基态结构下的总能量, $\varepsilon_{\text {HOMO }}$ 代表阴离子基态结构最 高占据轨道(HOMO)的本征值. 谱图拟合是将计算得到 的垂直电子逸出能值以高斯函数展开, 半峰宽为 0.10 $\mathrm{eV}$.

\section{3 结果与讨论}

\section{1 优化构型}

在 B3LYP/Basis-II 水平下, 我们发现 $\mathrm{NbMoS}_{n}^{-/ 0}$ $(n=3 \sim 7)$ 体系的势能面较为平缓, 除发现体系最稳构 型外, 还得到若干相对能量与之靠近 $(\leqslant 0.30 \mathrm{eV})$ 的异构
体(图 1 5). 对这些能量靠近的异构体, 我们采用更高 精度的耦合族 $[\operatorname{CCSD}(\mathrm{T})]$ 单点能计算方法来作进一步确 认，计算结果列于表 1 以及附录表 $\mathrm{S} 1$. 总的来说, B3LYP 下的结果与 $\operatorname{CCSD}(\mathrm{T})$ 单点能计算下的结果基本 一致，仅个别相对能量极其靠近的异构体之间发生相对 稳定性的翻转. 对这些发生翻转的体系, 我们将在文中 做进一步讨论. 其他在 B3LYP/Basis-I 低基组与 B3LYP/Basis-II 高基组水平下优化得到的结构与其相对 能量见于附录(图 $\mathrm{S} 1 \sim \mathrm{S} 11$ ).

\subsection{1 $\mathrm{NbMoS}_{3}$ 和 $\mathrm{NbMoS}_{3}^{-}$}

对 $\mathrm{NbMoS}_{3}^{-/ 0}$ 体系, 我们设计了各种可能的初始结 构, 包括两金属原子间键连一个桥硫、两个桥硫以及三 个桥硫的情况，对每种初始结构我们还考虑了它们在不 同自旋多重度下的情况. 计算结果表明, 在 B3LYP 水平 下得到的中性 $\mathrm{NbMoS}_{3}$ 最稳定构型是一个具有 $C_{s}$ 对称 性的四重态 $\left({ }^{4} \mathrm{~A}^{\prime}\right)$ 结构(图 1a), 该结构含有两个桥硫原子, 剩下的一个硫原子以端硫形式与铌原子相连. 除该最稳 构型外，我们还找到一个与之能量靠近的竞争异构体， 该异构体相对能量仅比最稳构型高了 $0.11 \mathrm{eV}$, 它是一 个具有 $C_{s}\left({ }^{2} \mathrm{~A}^{\prime}\right)$ 对称性(图 1b)且含有三个桥硫的结构. 此外, 与最稳定结构形似的二重态异构体 $\left(C_{s}{ }^{2} \mathrm{~A}^{\prime}\right.$, 图 1c), 其相对能量比最稳构型高了 $0.15 \mathrm{eV}$, 它的 $\mathrm{Nb}-\mathrm{Mo}$ 金属键长略有缩短变为 $2.414 \AA$. 我们对这三个竞争的 异构体采用更高精度的耦合簇 $[\mathrm{CCSD}(\mathrm{T})]$ 方法进行单点 能计算, 结果显示两桥一端的二重态异构体(图 1c)其相 对能量仍然较高, 但原先在 B3LYP 水平下次稳的三桥 构型(图 $1 \mathrm{~b}$ )在 $\operatorname{CCSD}(\mathrm{T})$ 单点能计算水平下却要比两桥一 端的四重态(图 1a)能量稍低, 低了 $0.09 \mathrm{eV}$. 在现有的计 算水平下, 我们认为该三桥的 $C_{s}\left({ }^{2} \mathrm{~A}^{\prime \prime}\right)$ 构型(图 1b)为中 性 $\mathrm{NbMoS}_{3}$ 的基态结构.

对于 $\mathrm{NbMoS}_{3}^{-}$阴离子, 其最稳定构型与中性基态 结构(图 1b)相类似，也是一个含有三个桥硫原子的闭壳

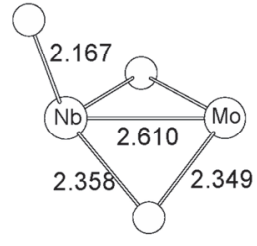

(a) $\mathrm{NbMoS}_{3}\left(C_{s}{ }^{4} \mathrm{~A} " 0.00 \mathrm{eV}\right)$

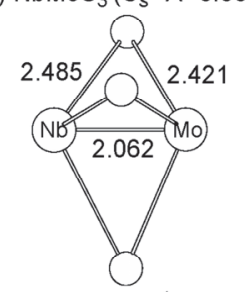

(d) $\mathrm{NbMoS}_{3}^{-}\left(C_{3 v}{ }^{1} \mathrm{~A}_{1} 0.00 \mathrm{eV}\right)$

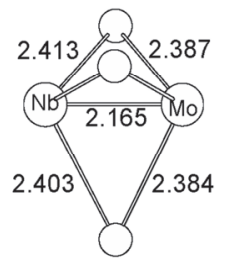

(b) $\operatorname{NbMoS}_{3}\left(C_{s}^{2} A^{\prime \prime} 0.11 \mathrm{eV}\right)$

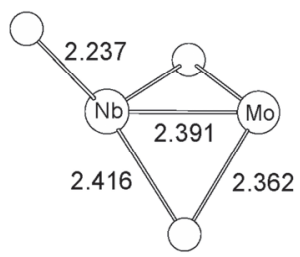

(e) $\mathrm{NbMoS}_{3}^{-}\left(C_{s}{ }^{3} \mathrm{~A}^{\prime \prime} 0.18 \mathrm{eV}\right)$

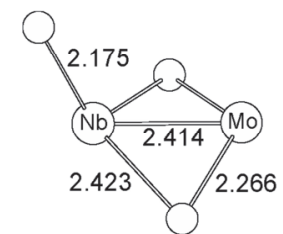

(c) $\mathrm{NbMoS}_{3}\left(C_{s}^{2} \mathrm{~A}^{\prime} 0.15 \mathrm{eV}\right)$

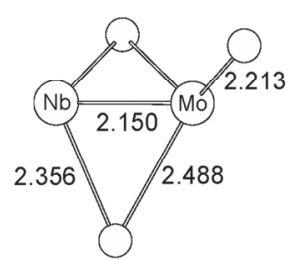

(f) $\mathrm{NbMoS}_{3}^{-}\left(C_{s}^{1} \mathrm{~A}^{\prime} 0.20 \mathrm{eV}\right)$

图 1 在 B3LYP/Basis-II 水平下得到的 $\mathrm{NbMoS}_{3}^{-/ 0}$ 体系最稳构型及与其相对能量靠近的异构体(键长单位为 $\AA$ )

Figure 1 Optimized structures for $\mathrm{NbMoS}_{3}^{-/ 0}$ at the B3LYP/Basis-II level of theory. The relative energies are in $\mathrm{eV}$ and the bond lengths are in angstroms 
表 $1 \mathrm{NbMoS}_{n}{ }^{-}(n=3 \sim 7)$ 在不同计算水平下的相对能量及其绝热电子逸出能(ADE)与第一点垂直电子逸出能 $\left(\mathrm{VDE}_{1 \mathrm{st}}\right)$ 的结果

Table 1 Relative energies of selected low-lying isomers of $\operatorname{NbMoS}_{n}^{-}(n=3 \sim 7)$ with different basis sets, and comparisons with those from the CCSD(T) single-point calculations at the B3LYP geometries together with the corresponding ADE and the first VDE values

\begin{tabular}{|c|c|c|c|c|c|}
\hline Cluster & B3LYP/Basis-I ${ }^{a, b}$ & B3LYP/Basis-II ${ }^{a, c}$ & $\operatorname{CCSD}(\mathrm{T})-\mathrm{SP}^{a, d}$ & $\mathrm{ADE}^{a}$ & $\mathrm{VDE}_{1 \mathrm{st}}{ }^{a}$ \\
\hline $\mathrm{NbMoS}_{3}^{-}\left(C_{3 v}{ }^{1} \mathrm{~A}_{1}\right)$ & 0.00 & 0.00 & 0.00 & 2.54 & 2.87 \\
\hline $\operatorname{NbMoS}_{3}^{-}\left(C_{s}^{3} \mathrm{~A}^{\prime \prime}\right)$ & 0.10 & 0.18 & 0.48 & 2.36 & 2.65 \\
\hline $\mathrm{NbMoS}_{3}^{-}\left(C_{s}{ }^{1} \mathrm{~A}^{\prime}\right)$ & 0.15 & 0.20 & 0.35 & 2.35 & 3.01 \\
\hline $\mathrm{NbMoS}_{4}^{-}\left(C_{s}^{3} \mathrm{~A}^{\prime \prime}\right)$ & 0.00 & 0.00 & 0.13 & 2.93 & 3.33 \\
\hline $\mathrm{NbMoS}_{4}^{-}\left(C_{s}{ }^{5} \mathrm{~A}^{\prime \prime}\right)$ & 0.02 & 0.03 & 0.24 & 2.90 & 3.75 \\
\hline $\mathrm{NbMoS}_{4}^{-}\left(C_{s}{ }^{5} \mathrm{~A}^{\prime \prime}\right)$ & 0.04 & 0.05 & 0.25 & 2.88 & 3.80 \\
\hline $\mathrm{NbMoS}_{4}^{-}\left(C_{3 v}{ }^{3} \mathrm{~A}_{1}\right)$ & 0.25 & 0.24 & 0.26 & 2.69 & 3.07 \\
\hline $\mathrm{NbMoS}_{4}^{-}\left(C_{s}^{3} \mathrm{~A}^{\prime}\right)$ & 0.26 & 0.27 & 0.43 & 2.66 & 2.97 \\
\hline $\mathrm{NbMoS}_{4}^{-}\left(C_{s}{ }^{1} \mathrm{~A}^{\prime}\right)$ & 0.30 & 0.30 & 0.00 & 2.63 & 2.81 \\
\hline $\mathrm{NbMoS}_{5}^{-}\left(C_{s}{ }^{3} \mathrm{~A}^{\prime}\right)$ & 0.00 & 0.00 & 0.19 & 3.49 & 3.82 \\
\hline $\operatorname{NbMoS}_{5}^{-}\left(C_{s}{ }^{1} \mathrm{~A}^{\prime}\right)$ & 0.05 & 0.06 & 0.00 & 3.43 & 3.68 \\
\hline $\mathrm{NbMoS}_{5}^{-}\left(C_{s}{ }^{3} \mathrm{~A}^{\prime \prime}\right)$ & 0.21 & 0.18 & 0.26 & 3.31 & 3.73 \\
\hline $\operatorname{NbMoS}_{5}^{-}\left(C_{s}{ }^{1} \mathrm{~A}^{\prime}\right)$ & 0.22 & 0.23 & 0.34 & 3.25 & 3.95 \\
\hline $\operatorname{NbMoS}_{5}^{-}\left(C_{s}^{3} \mathrm{~A}^{\prime}\right)$ & 0.23 & 0.24 & 0.46 & 3.24 & 3.84 \\
\hline $\mathrm{NbMoS}_{6}^{-}\left(C_{s}{ }^{1} \mathrm{~A}^{\prime}\right)$ & 0.00 & 0.00 & 0.00 & 4.27 & 4.69 \\
\hline $\operatorname{NbMoS}_{7}^{-}\left(C_{1}{ }^{1} \mathrm{~A}\right)$ & 0.00 & 0.00 & 0.00 & 4.13 & 4.46 \\
\hline $\mathrm{NbMoS}_{7}^{-}\left(C_{1}{ }^{1} \mathrm{~A}\right)$ & 0.13 & 0.15 & 0.11 & 3.98 & 4.48 \\
\hline
\end{tabular}

${ }^{a}$ All energies are in $\mathrm{eV} ;{ }^{b} \mathrm{~B} 3 \mathrm{LYP} / \mathrm{Basis}-\mathrm{I} ;{ }^{c} \mathrm{~B} 3 \mathrm{LYP} / \mathrm{Basis}-\mathrm{II} ;{ }^{d} \mathrm{CCSD}(\mathrm{T})$ single-point calculations at the B3LYP/Basis-II geometries.

层高对称性结构 $\left(C_{3 v}{ }^{1} \mathrm{~A}_{1}\right.$, 图 $\left.1 \mathrm{~d}\right)$. 对于端硫键连在铌原 子上的二桥结构 $\left(C_{s}{ }^{3} \mathrm{~A} "\right.$, 图 1e), 其相对能量比最稳构 型高了 $0.18 \mathrm{eV}$, 而端硫位于钼原子上的二桥结构 $\left(C_{s}\right.$ ${ }^{1} \mathrm{~A}$ ', 图 1f), 其相对能量比最稳构型高了 $0.20 \mathrm{eV}$. 对这 些异构体我们也采用了 $\operatorname{CCSD}(\mathrm{T})$ 方法进行单点能计算, 结果与 B3LYP 下的结论相一致.

\subsection{2 $\mathrm{NbMoS}_{4}$ 和 $\mathrm{NbMoS}_{4}^{-}$}

在 $\mathrm{NbMoS}_{3}^{-10}$ 各种稳定构型的基础上，我们考虑新 增一个硫原子的不同摆放位置。计算结果表明, $\mathrm{NbMoS}_{4}^{-/ 0}$ 体系的势能面也比较平缓, 除最稳构型外, 也发现多个能量与之靠近的异构体. 其中 $\mathrm{NbMoS}_{4}$ 中性 的最稳定构型是一个对称性为 $C_{s}\left({ }^{2} \mathrm{~A}^{\prime}\right)$ 的二重态结构(图 2a). 该结构含有两个桥硫及两个分别位于铌和钼原子 上的端硫原子. 我们还得到一个相对能量仅比最稳构型 高了 $0.01 \mathrm{eV}$ 的异构体 $\left(C_{s}{ }^{2} \mathrm{~A} "\right.$, 图 $\left.2 \mathrm{~b}\right)$, 这两个异构体不 仅结构上类似, 相对能量上也极其接近, 仅在电子态上 有所不同 $\left({ }^{2} \mathrm{~A}^{\prime \prime} \mathrm{vs}{ }^{2} \mathrm{~A}^{\prime}\right)$, 即使通过 $\operatorname{CCSD}(\mathrm{T})$ 单点能计算也 很难将其完全区分开, 所以在实验中这两种异构体都有 可能被观测到. 此外, 有一个两桥两端的异构体 $\left(C_{s}{ }^{2} \mathrm{~A}^{\prime}\right.$, 图 2c), 该异构体的两个金属原子与两个桥硫原子共同 组成一个近平面的 $\mathrm{NbMoS}_{2}$ 四元环结构, 剩下的两个端 硫指向该四元环的不同方向, 其相对能量比最稳构型高 了 $0.19 \mathrm{eV}$. 还有三个三桥一端的异构体 (图 $2 \mathrm{~d} \sim 2 \mathrm{~g}$ ), 其 相对能量均比最稳构型高了约 $0.29 \mathrm{eV}$.

对于 $\mathrm{NbMoS}_{4}^{-}$阴离子的最稳构型(图 2h), 其几何结 构与中性最稳定结构(图 $2 \mathrm{a}$ )类似, 是一个含有两个桥硫
和两个端硫, 对称性为 $C_{s}$ 的三重态 $\left({ }^{3} \mathrm{~A} "\right)$ 结构. 另有两个 五重态的两桥两端结构(图 $2 \mathrm{i}$ 和 $2 \mathrm{j}$ ), 其相对能量仅比最 稳结构分别高了 0.03 和 $0.05 \mathrm{eV}$. 此外, 我们还得到了三 个相对能量分别为 $0.24 、 0.27$ 和 $0.30 \mathrm{eV}$ 的异构体(图 $2 \mathrm{k} \sim 2 \mathrm{~m})$. 在这三个异构体中, 有一个与最稳构型结构 类似的一重态 $\left(C_{s}{ }^{1} \mathrm{~A}\right.$ ', 图 $\left.2 \mathrm{~m}\right)$, 其相对能量虽比最稳构 型高了 $0.30 \mathrm{eV}$, 但该异构体在 $\operatorname{CCSD}(\mathrm{T})$ 单点能计算中 被认为是最稳构型, 相对能量比 B3LYP 水平下的最稳 构型低了 $0.13 \mathrm{eV}$. 对这两个稳定性发生翻转的异构体, 我们在 B3LYP 水平下计算了它们的红外光谱数据, 结 果发现 B3LYP 水平下的最稳构型(图 2h) 在频率为 512.0 $\mathrm{cm}^{-1}(\mathrm{~s})$ 和 $501.65 \mathrm{~cm}^{-1}(\mathrm{vs})$ 处有两个强峰, 分别对应端硫 的对称伸缩振动 $\left(v_{\mathrm{s}}\right)$ 和反对称伸缩振动 $\left(v_{\mathrm{as}}\right)$. 另外, 在频 率 $431.6 \mathrm{~cm}^{-1}$ (s)处有一个较强的峰, 在 $404.1 \mathrm{~cm}^{-1}(\mathrm{~m})$ 处有一个中等强度的峰, 分别对应桥硫的反对称伸缩振 动 $\left(v_{\mathrm{as}}\right)$ 和对称伸缩振动 $\left(v_{\mathrm{s}}\right)$. 而另一个在 $\operatorname{CCSD}(\mathrm{T})$ 单点能 计算中被认为最稳定的结构(图 3m), 计算发现其在振 动频率 $506.7 \mathrm{~cm}^{-1}(\mathrm{vs})$ 和 $493.7 \mathrm{~cm}^{-1}(\mathrm{vs})$ 处有两个对应端 硫的对称伸缩振动 $\left(v_{\mathrm{s}}\right)$ 和反对称伸缩振动 $\left(v_{\mathrm{as}}\right)$ 的强峰. 在 $461.5 \mathrm{~cm}^{-1}(\mathrm{~m})$ 处有一中等强度的峰, 在 $432.1 \mathrm{~cm}^{-1}(\mathrm{vw})$ 处有一非常弱的峰, 分别对应桥硫的反对称伸缩振动 $\left(v_{\mathrm{as}}\right)$ 和对称伸缩振动 $\left(v_{\mathrm{s}}\right)$. (文中振动强度表示符号: $\mathrm{w}$ 弱; $\mathrm{vw}$ 极弱; $\mathrm{m}$ 中; $\mathrm{s}$ 强; vs 极强)

\subsection{3 $\mathrm{NbMoS}_{5}$ 和 $\mathrm{NbMoS}_{5}^{-}$}

在 $\mathrm{NbMoS}_{4}^{-} / 0$ 稳定构型基础上, 我们继续对其添加 硫原子. 最终优化得到具有两桥三端的中性 $\mathrm{NbMoS}_{5}$ 最 


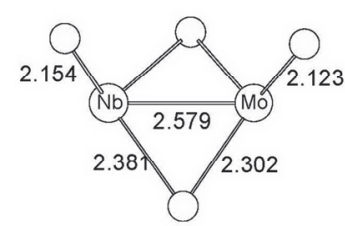

(a) $\mathrm{NbMoS}_{4}\left(C_{s}{ }^{2} \mathrm{~A}^{\prime} 0.00 \mathrm{eV}\right)$

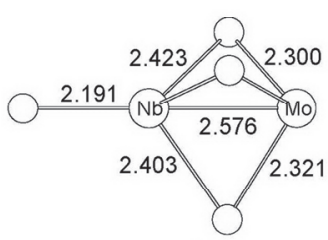

(e) $\mathrm{NbMoS}_{4}\left(\mathrm{C}_{s}{ }^{2} \mathrm{~A} " 0.288 \mathrm{eV}\right)$

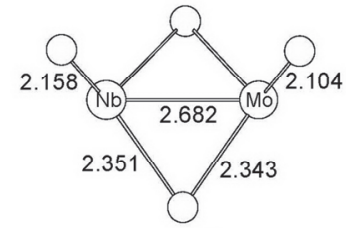

(b) $\mathrm{NbMoS}_{4}\left(C_{s}{ }^{2} \mathrm{~A} " 0.01 \mathrm{eV}\right)$

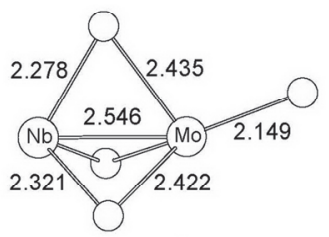

(f) $\mathrm{NbMoS}_{4}\left(C_{s}{ }^{2} A^{\prime \prime} 0.291 \mathrm{eV}\right)$

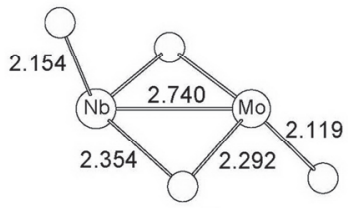

(c) $\mathrm{NbMoS}_{4}\left(C_{\mathrm{s}}{ }^{2} \mathrm{~A}^{\prime} 0.19 \mathrm{eV}\right)$

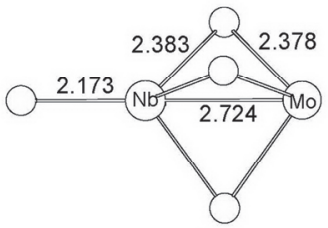

(d) $\mathrm{NbMoS}_{4}\left(C_{3 v}{ }^{4} \mathrm{~A}_{1} 0.24 \mathrm{eV}\right)$

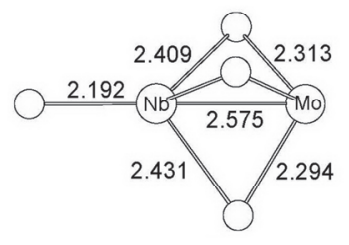

(g) $\mathrm{NbMoS}_{4}\left(C_{s}{ }^{2} A^{\prime} 0.293 \mathrm{eV}\right)$

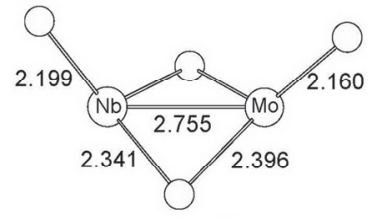

(h) $\mathrm{NbMoS}_{4}^{-}\left(C_{s}{ }^{3} \mathrm{~A}^{\prime \prime} 0.00 \mathrm{eV}\right)$

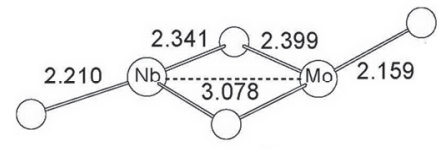

(i) $\mathrm{NbMoS}_{4}^{-}\left(C_{s}{ }^{5} \mathrm{~A}^{\prime \prime} 0.03 \mathrm{eV}\right)$

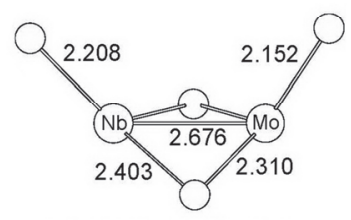

(I) $\mathrm{NbMoS}_{4}{ }^{-}\left(C_{s}{ }^{3} \mathrm{~A}^{\prime} 0.27 \mathrm{eV}\right)$

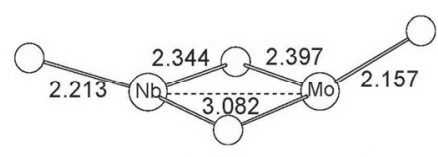

(j) $\mathrm{NbMoS}_{4}{ }^{-}\left(C_{\mathrm{s}}{ }^{5} \mathrm{~A}^{\prime \prime} 0.05 \mathrm{eV}\right)$

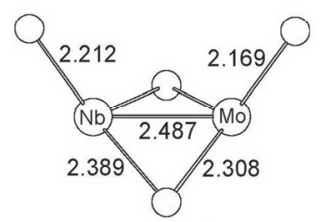

(m) $\mathrm{NbMoS}_{4}^{-}\left(C_{s}{ }^{1} A^{\prime} 0.30 \mathrm{eV}\right)$

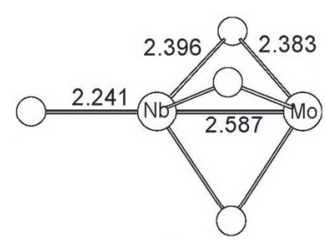

(k) $\mathrm{NbMoS}_{4}^{-}\left(C_{3 v}{ }^{3} \mathrm{~A}_{1} 0.24 \mathrm{eV}\right)$

图 2 在 B3LYP/Basis-II 水平下得到的 $\mathrm{NbMoS}_{4}^{-/ 0}$ 体系最稳构型及与其相对能量靠近的异构体(键长单位为 $\AA$ )

Figure 2 Optimized structures for $\mathrm{NbMoS}_{4}^{-/ 0}$ at the B3LYP/Basis-II level of theory. The relative energies are in eV and the bond lengths are in angstroms

稳定构型 $\left(C_{s}{ }^{2} \mathrm{~A}^{\prime}\right.$, 图 3a). 其中三个端硫原子, 一个位于 铌原子, 两个位于钼原子上. 该结构可视作在中性 $\mathrm{NbMoS}_{4}$ 基础上于钼原子添加一个端硫原子而来. 同时 我们也得到一个相对能量为 $0.19 \mathrm{eV}$ 具有 $C_{s}\left({ }^{2} \mathrm{~A}^{\prime}\right)$ 对称 性的三桥构型异构体(图 3b), 该结构中的 $\mathrm{Nb}-\mathrm{Mo}$ 金属 键长 $(2.718 \AA)$ 比二桥结构的最稳构型 (3.055 $\AA$ ) 要短得 多. 这两个异构体在 $\operatorname{CCSD}(\mathrm{T})$ 单点能计算水平下的结论 支持它们在 B3LYP 计算水平下的结果.

对 $\mathrm{NbMoS}_{5}^{-}$阴离子, 其体系势能面较为平缓, 有多 个与最稳构型相对能量靠近的异构体. 计算结果显示 $\mathrm{NbMoS}_{5}^{-}$的最稳定构型与中性的最稳定构型类似, 也 是一个具有 $C_{s}\left({ }^{3} \mathrm{~A}^{\prime}\right)$ 对称性的双桥结构(图 3c), 该结构对 应的一重态 $\left(C_{s}{ }^{1} \mathrm{~A}^{\prime}\right)$, 其相对能量仅比最稳构型高了 0.06 $\mathrm{eV}$ (图 3d), 该结构中的三个端硫, 一个键连在金属铌 上, 两个与金属钼相连. 该构型与最稳构型相比, 其金 属一金属间距离更短, 距离为 $2.823 \AA$. 如果双桥结构的 $\mathrm{NbMoS}_{5}^{-}$异构体中, 剩下的三个端硫原子有两个位于 铌, 一个位于钿上, 则其一重态和三重态的相对能量要 比最稳构型分别高了 0.23 和 $0.24 \mathrm{eV}$ (图 3f 和 3g). 此外,
我们还找到一个三桥结构的异构体(图 3e), 其相对能量 比最稳构型高了 $0.18 \mathrm{eV}$. 对这些异构体我们都采用 $\operatorname{CCSD}(\mathrm{T})$ 方法进行单点能计算(表 1), 其结果与 B3LYP 水平下的结果基本一致, 仅相对能量最为靠近的两个异 构体(图 3c 和 3d)的稳定性发生颠倒, 即认为一重态的双 桥结构异构体(图 3d)比其三重态异构体(图 3c)更加稳定. 对于这两个 $\mathrm{NbMoS}_{5}^{-}$阴离子相对能量发生翻转的异构 体, 我们同样对其红外光谱进行了模拟计算, 结果发现 三重态的异构体(图 4c)在 $536.3 \mathrm{~cm}^{-1}$ (s) 处有一强峰, 对 应端硫的反对称伸缩振动 $\left(v_{\mathrm{as}}\right)$. 在 $520.4 \mathrm{~cm}^{-1}(\mathrm{vw})$ 处有 一非常弱的峰, 在 $505.2 \mathrm{~cm}^{-1}$ (vs) 处有一非常强的峰, 对应端硫的对称伸缩振动 $\left(v_{\mathrm{s}}\right)$. 其中在 $520.4 \mathrm{~cm}^{-1}(\mathrm{vw})$ 处的峰由于其峰强非常弱, 所以在实验中可能会淹没于 两旁的两个强峰中, 成为鉴别该异构体的一个明显指纹 特征. 在 $436.7 \mathrm{~cm}^{-1}(\mathrm{~m})$ 和 $402.6 \mathrm{~cm}^{-1}(\mathrm{w})$ 处分别有一中 等强度和较弱的峰, 对应桥硫的反对称伸缩振动 $\left(v_{\mathrm{as}}\right)$ 和 对称伸缩振动 $\left(v_{\mathrm{s}}\right.$ ). 对于另一个单重态异构体(图 $4 \mathrm{~d}$ ), 其 红外光谱模拟计算发现: 在 $530.8 \mathrm{~cm}^{-1}$ (s) 处有一强峰, 对应端硫的反对称伸缩振动 $\left(v_{\mathrm{as}}\right)$. 在 $515.7 \mathrm{~cm}^{-1}(\mathrm{~m})$ 和 


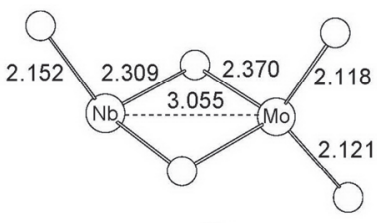

(a) $\mathrm{NbMoS}_{5}\left(C_{s^{2}} \mathrm{~A}^{\prime} 0.00 \mathrm{eV}\right)$

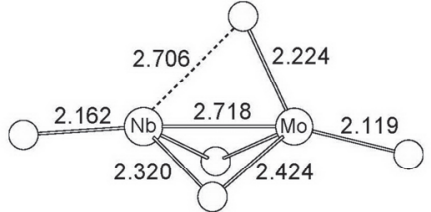

(b) $\mathrm{NbMoS}_{5}\left(C_{s}{ }^{2} \mathrm{~A}^{\prime} 0.19 \mathrm{eV}\right)$

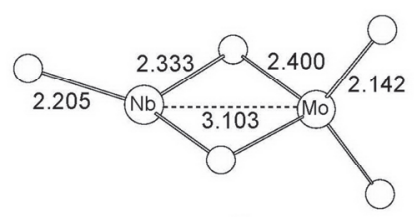

(c) $\mathrm{NbMoS}_{5}{ }^{-}\left(\mathrm{C}^{3}{ }^{3} \mathrm{~A}^{\prime} 0.00 \mathrm{eV}\right)$

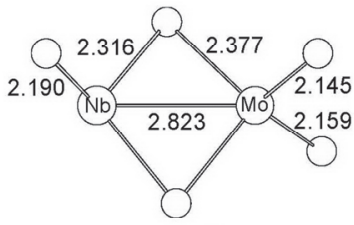

(d) $\mathrm{NbMoS}_{5}^{-}\left(C_{s}{ }^{1} \mathrm{~A}^{\prime} 0.06 \mathrm{eV}\right)$

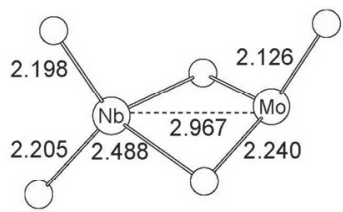

(f) $\mathrm{NbMoS}_{5}{ }^{-}\left(C_{s}{ }^{1} \mathrm{~A}^{\prime} 0.23 \mathrm{eV}\right)$

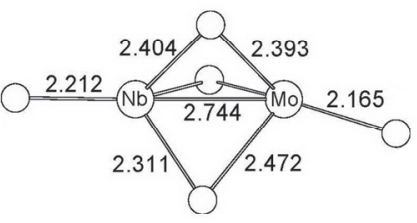

(e) $\mathrm{NbMoS}_{5}{ }^{-}\left(C_{s}{ }^{3} \mathrm{~A}^{\prime \prime} 0.18 \mathrm{eV}\right)$

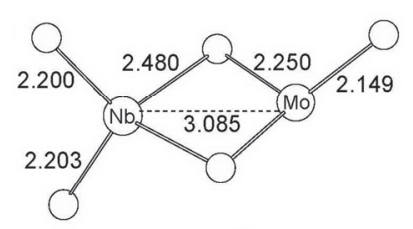

(g) $\mathrm{NbMoS}_{5}{ }^{-}\left(C_{s}{ }^{3} \mathrm{~A}^{\prime} 0.24 \mathrm{eV}\right)$

图 3 在 B3LYP/Basis-II 水平下得到的 $\mathrm{NbMoS}_{5}^{-10}$ 体系最稳构型及与其相对能量靠近的异构体(键长单位为 $\AA$ )

Figure 3 Optimized structures for $\mathrm{NbMoS}_{5}^{-/ 0}$ at the B3LYP/Basis-II level of theory. The relative energies are in eV and the bond lengths are in angstroms

$501.4 \mathrm{~cm}^{-1}$ (vs)处分别有一个中等强度和非常强的峰, 对应端硫的对称伸缩振动 $\left(v_{\mathrm{s}}\right)$. 在 $447.1 \mathrm{~cm}^{-1}(\mathrm{~m})$ 和 $419.8 \mathrm{~cm}^{-1}(\mathrm{w})$ 处分别有一中等强度和较弱的峰, 对应 桥硫的反对称伸缩振动 $\left(v_{\mathrm{as}}\right)$ 和对称伸缩振动 $\left(v_{\mathrm{s}}\right)$. 上述谱 峰位置与 Andew 小组 ${ }^{[44,45]}$ 对 $\mathrm{NbS}_{x} 、 \mathrm{MoS}_{x}$ 团簇的矩阵红 外光谱实验中, 所报道的端硫振动频率的理论值与实验 值基本相符 $\left(\mathrm{NbS}_{2}\right.$ 端硫的 $v_{\mathrm{s}}$ 和 $v_{\mathrm{as}}$ 理论值分别为 536.9 $\mathrm{cm}^{-1}$ 和 $534.8 \mathrm{~cm}^{-1}$, 其实验值分别为 $525.1 \mathrm{~cm}^{-1}$ 和 531.0 $\mathrm{cm}^{-1} ; \mathrm{MoS}_{2}$ 端硫的 $v_{\mathrm{s}}$ 和 $v_{\mathrm{as}}$ 理论值分别 $523.8 \mathrm{~cm}^{-1}$ 和 $534.8 \mathrm{~cm}^{-1}, v_{\mathrm{as}}$ 实验值为 $535.7 \mathrm{~cm}^{-1}$ ).

\subsection{4 $\mathrm{NbMoS}_{6}$ 和 $\mathrm{NbMoS}_{6}^{-}$}

对中性 $\mathrm{NbMoS}_{6}$ 的计算结果发现, 其最稳构型为一 个具有 $C_{s}\left({ }^{2} \mathrm{~A}^{\prime}\right)$ 对称性的二重态(图 4a), 它含有两个端 硫、两个桥硫和一个桥连的二硫 $\left(\mathrm{S}_{2}\right)$ 基团，该二硫 $\left(\mathrm{S}_{2}\right)$ 基 团中 $\mathrm{S}-\mathrm{S}$ 键键长为 $2.126 \AA$, 与相同基组水平下计算得 到的游离态的 $\mathrm{S}_{2}^{2-}\left({ }^{1} \Sigma_{\mathrm{g}}^{+}\right)$的键长 $(2.180 \AA)$ 相近, 可以 看作由 $\mathrm{S}_{2}^{2-}$ 基团与 $\mathrm{NbMoS}_{4}^{2+}$ 相结合得到. 此外, 我们还 得到一个二桥四端结构的异构体 $\left(C_{s}{ }^{2} \mathrm{~A}^{\prime}\right.$, 图 $\left.4 \mathrm{~b}\right)$, 其相对 能量为 $0.20 \mathrm{eV}$. 这两个异构体在 $\operatorname{CCSD}(\mathrm{T})$ 水平下计算 单点能, 其结果与 B BLYP 水平下的结果一致, 支持含 $\mathrm{S}_{2}^{2-}$ 基团的异构体(图 4a)为中性 $\mathrm{NbMoS}_{6}$ 的基态.

对于 $\mathrm{NbMoS}_{6}^{-}$阴离子, 计算结果显示最稳构型为 闭壳层的两桥四端构型 $\left(C_{s}{ }^{1} \mathrm{~A}^{\prime}\right.$, 图 4c), 该结构与中性基 态的构型差异较大. 可能是由于多余的一个电子加入 $\mathrm{S}_{2}^{2-}$ 基团中, 导致该基团断裂为两个端硫的缘故.

\subsection{5 $\mathrm{NbMoS}_{7}$ 和 $\mathrm{NbMoS}_{7}^{-}$}

对富硫类型的 $\mathrm{NbMoS}_{7}^{-/ 0}$ 体系, 计算结果显示, 中 性 $\mathrm{NbMoS}_{7}$ 的最稳构型是一个含两个桥硫、三个端硫和 一个端连在金属铌原子位上的二硫 $\left(\mathrm{S}_{2}\right)$ 基团的结构(图 $5 \mathrm{a})$ ，通过自旋密度分析得知 $\left(\mathrm{S}_{2}\right)$ 基团上分布有一个单电 子, 且 $\mathrm{S}-\mathrm{S}$ 键长 $(2.007 \AA)$ 与相同基组水平下计算得到 的游离态 $\mathrm{S}_{2}^{-}\left({ }^{2} \Pi_{\mathrm{g}}\right)$ 的键长 $(2.029 \AA)$ 相接近, 因此可以看 作以 $\left(\mathrm{S}_{2}^{-}\right)$基团与 $\mathrm{NbMoS}_{5}^{+}$单元相结合得到. 同时我们 还得到了一个相对能量为 $0.04 \mathrm{eV}$, 对称性为 $C_{s}\left({ }^{2} \mathrm{~A} "\right)$ 的 二重态异构体(图 5b), 该异构体含有一个位于金属铌原 子上的端位 $\mathrm{S}_{3}$ 基团, 其 $\mathrm{S}-\mathrm{S}$ 键长为 $2.117 \AA$. 此外我们 还找到一个相对能量为 $0.23 \mathrm{eV}$ 的构型(图 5c), 该构型 含有两种不同类型的 $\mathrm{S}_{2}$ 基团，即一个桥连的 $\mathrm{S}_{2}^{2-}$ 和一个 端连的 $\mathrm{S}_{2}^{2-}$ 基团. 对这些异构体，我们亦通过 $\operatorname{CCSD}(\mathrm{T})$ 单点能计算的方法来确认其相对稳定性, 结果发现 B3LYP 方法下的最稳结构(图 5a) 与次稳构型(图 5b)的相 对能量仍非常靠近，仅相差 $0.06 \mathrm{eV}$, 其中含 $\mathrm{S}_{3}$ 基团的次 稳构型(图 5b)相对能量略低, 理论模拟与实验谱图的对 照有利于进一步辨别哪种构型是真正的基态结构.

计算结果指出 $\mathrm{NbMoS}_{7}^{-}$阴离子的最稳定构型是一 个具有 $C_{1}$ 对称性的含有两个桥硫三个端硫和一个端连 在铌原子上的 $\mathrm{S}_{2}$ 基团的结构(图 5d). 该结构中的 $\mathrm{S}_{2}$ 基团 的 $\mathrm{S}-\mathrm{S}$ 键长为 $2.096 \AA$, 与相同水平下计算得到的游离 态 $\mathrm{S}_{2}^{2-}$ 的键长 $(2.180 \AA)$ 接近, 因此也可以看作是以 $\mathrm{S}_{2}^{2-}$ 基团与 $\mathrm{NbMoS}_{5}^{+}$相结合得到, 该构型与中性 $\mathrm{NbMoS}_{7}$ 在 B3LYP 水平下的最稳定构型类似. 此外我们也得到了 


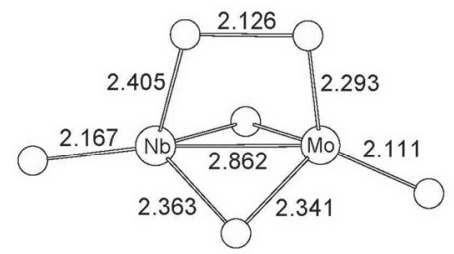

(a) $\mathrm{NbMoS}_{6}\left(C_{\mathrm{s}}{ }^{2} \mathrm{~A}^{\prime} 0.00 \mathrm{eV}\right)$

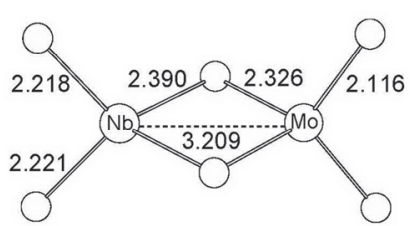

(b) $\mathrm{NbMoS}_{6}\left(\mathrm{C}_{\mathrm{s}}{ }^{2} \mathrm{~A}^{\prime} 0.20 \mathrm{eV}\right)$

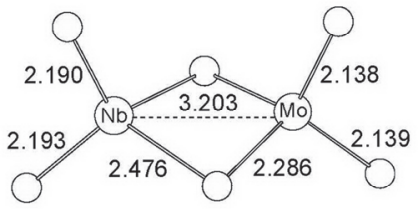

(c) $\mathrm{NbMoS}_{6}^{-}\left(\mathrm{C}_{\mathrm{s}}{ }^{1} \mathrm{~A}^{\prime} 0.00 \mathrm{eV}\right)$

图 4 在 B3LYP/Basis-II 水平下得到的 $\mathrm{NbMoS}_{6}^{-/ 0}$ 体系最稳构型及与其相对能量靠近的异构体(键长单位为 $\AA$ )

Figure 4 Optimized structures for $\mathrm{NbMoS}_{6}^{-10}$ at the B3LYP/Basis-II level of theory. The relative energies are in $\mathrm{eV}$ and the bond lengths are in angstroms

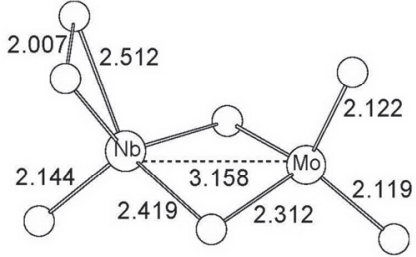

(a) $\operatorname{NbMoS}_{7}\left(C_{s}^{2} \mathrm{~A}^{\prime \prime} 0.00 \mathrm{eV}\right)$

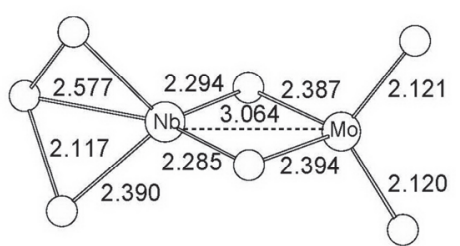

(b) $\mathrm{NbMoS}_{7}\left(C_{s}{ }^{2} \mathrm{~A}^{\prime} 0.04 \mathrm{eV}\right)$

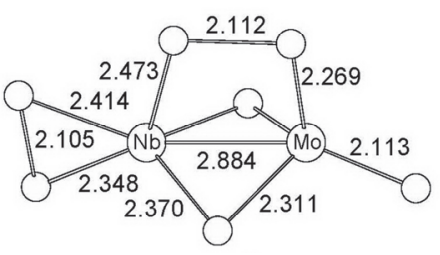

(c) $\operatorname{NbMoS}_{7}\left(C_{s}^{2} A^{\prime} 0.23 \mathrm{eV}\right)$

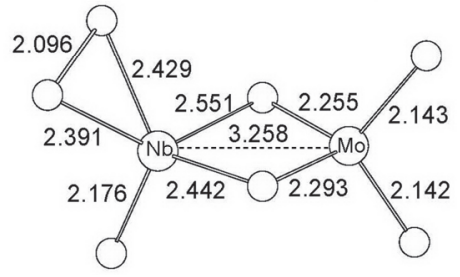

(d) $\mathrm{NbMoS}_{7}^{-}\left(\mathrm{C}_{1}{ }^{1} \mathrm{~A} 0.00 \mathrm{eV}\right)$

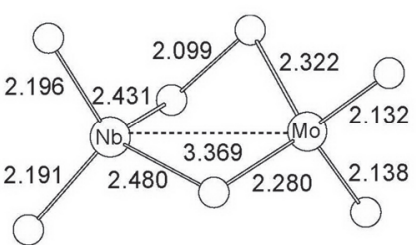

(e) $\operatorname{NbMoS}_{7}^{-}\left(C_{1}{ }^{1} \mathrm{~A} 0.15 \mathrm{eV}\right)$

图 5 在 B3LYP/Basis-II 水平下得到的 $\mathrm{NbMoS}_{7}^{-/ 0}$ 体系基态和与其相对能量靠近的异构体(键长单位为 $\AA$ )

Figure 5 Optimized structures for $\mathrm{NbMoS}_{7}^{-/ 0}$ at the B3LYP/Basis-II level of theory. The relative energies are in eV and the bond lengths are in angstroms

一个相对能量为 $0.15 \mathrm{eV}$ 含有四端两桥(两桥中一个为桥 连的 $\mathrm{S}_{2}$ 基团)的构型(图 5e). 这两个异构体我们也采用 更高精度的耦合簇 $[\operatorname{CCSD}(\mathrm{T})]$ 方法计算单点能, 得到的 结果与 B3LYP 水平下的结果相一致.

\section{2 光电子能谱模拟与分子轨道分析}

本工作计算了 $\mathrm{NbMoS}_{n}^{-}(n=3 \sim 7)$ 阴离子基态及与 之相对能量靠近的异构体的垂直电子逸出能 (VDEs), 采用广义 Koopmans 定理(GKT)加修正项的方法, 模拟 各个 $\mathrm{NbMoS}_{n}^{-}$体系的阴离子光电子能谱(PES), 模拟的 光电子谱图如图 6 所示; 各 $\mathrm{NbMoS}_{n}^{-}$体系对应第一点 $\mathrm{VDE}$ 值 $\left(\mathrm{VDE}_{1 \mathrm{st}}\right)$ 见表 1; 各基态构型的自旋电子密度图 见图 7; 各 $\mathrm{NbMoS}_{n}^{-10}(n=3 \sim 7)$ 体系有关的价层分子轨 道见图 8〜12. 这些图中黄色球表示硫原子, 红色球表 示铌原子, 天蓝色球表示钼原子.

\subsection{1 $\mathrm{NbMoS}_{3}$ 和 $\mathrm{NbMoS}_{3}{ }^{-}$}

中性 $\mathrm{NbMoS}_{3}$ 和阴离子 $\mathrm{NbMoS}_{3}^{-}$的价层分子轨道如 (图 8)所示. 中性基态 $\mathrm{NbMoS}_{3}\left(C_{s}{ }^{2} \mathrm{~A}^{\prime \prime}\right)$ 的价电子组态 为 $\cdots\left(12 a^{\prime \prime}\right)^{2}\left(23 a^{\prime}\right)^{2}\left(13 a^{\prime \prime}\right)^{2}\left(14 a^{\prime \prime}\right)^{1}\left(24 a^{\prime}\right)^{2}$. 其 HOMO 轨道 $\left(24 \mathrm{a}^{\prime}\right)^{2}$ 主要是两个金属间的 $4 \mathrm{~d}-\pi$ 成键轨道; 次外层是一
个单占轨道 $\left(14 \mathrm{a}^{\prime \prime}\right)^{1}$, 主要也是两个金属间的 $4 \mathrm{~d}-\pi$ 成键轨 道. 阴离子基态 $\mathrm{NbMoS}_{3}^{-}\left(C_{3 v}{ }^{1} \mathrm{~A}_{1}\right)$ 可认为是在中性 $\mathrm{NbMoS}_{3}$ 基态上增加一个电子得到, 其价电子组态是 $\cdots$ $(11 \mathrm{e})^{4}\left(12 \mathrm{a}_{1}\right)^{2}\left(2 \mathrm{a}_{2}\right)^{2}(12 \mathrm{e})^{4}$. 最高占据分子轨道 HOMO 的 $(12 \mathrm{e})^{4}$ 为全满的简并轨道, 主要是金属铌钼间的 $4 \mathrm{~d}-\pi$ 成 键轨道; 其次外层 $\left(2 \mathrm{a}_{2}\right)^{2}$ 主要成分为三个桥硫原子的 $3 \mathrm{p}$ 非键轨道; $\left(12 \mathrm{a}_{1}\right)^{2}$ 是金属间以 “头碰头” 形式形成 $4 \mathrm{~d}-\sigma$ 成键轨道. 打掉最外层 $(12 \mathrm{e})^{4}$ 简并轨道上的一个电子得 到第一点 $\operatorname{VDE}$ 值 $2.87 \mathrm{eV}$ (表 1), 对应模拟光电子谱图 中第一个谱峰 X 的位置(图 6a).

\subsection{2 $\mathrm{NbMoS}_{4}$ 和 $\mathrm{NbMoS}_{4}^{-}$}

中性基态 $\mathrm{NbMoS}_{4}\left(C_{s}{ }^{2} \mathrm{~A}^{\prime \prime}\right)$ 的价电子组态为 $\cdots\left(14 a^{\prime \prime}\right)^{2}\left(29 a^{\prime}\right)^{2}\left(30 a^{\prime}\right)^{2}\left(15 a^{\prime \prime}\right)^{2}\left(16 a^{\prime \prime}\right)^{1}$. 其中最高单占据分 子轨道 SOMO $\left(16 \mathrm{a}^{\prime \prime}\right)^{1}$ 主要为两金属原子上的 $4 \mathrm{~d}$ 轨道成 分; $\left(15 \mathrm{a}^{\prime \prime}\right)^{2}$ 主要是端硫和桥硫上的 $3 \mathrm{p}$ 轨道成分; 另外 $\left(30 \mathrm{a}^{\prime}\right)^{2}$ 轨道主要是金属铌与金属钿以 “头碰头” 形成的 $4 \mathrm{~d}-\sigma$ 成键轨道成分. 基态阴离子 $\mathrm{NbMoS}_{4}^{-}\left(C_{s}{ }^{1} \mathrm{~A}^{\prime}\right)$ 与中 性基态构型类似，也为两桥两端的结构. 其价电子组态 是 $\cdots\left(14 \mathrm{a}^{\prime \prime}\right)^{2}\left(29 \mathrm{a}^{\prime}\right)^{2}\left(30 \mathrm{a}^{\prime}\right)^{2}\left(15 \mathrm{a}^{\prime \prime}\right)^{2}\left(31 \mathrm{a}^{\prime}\right)^{2}$ (图 9c). 其中最外 

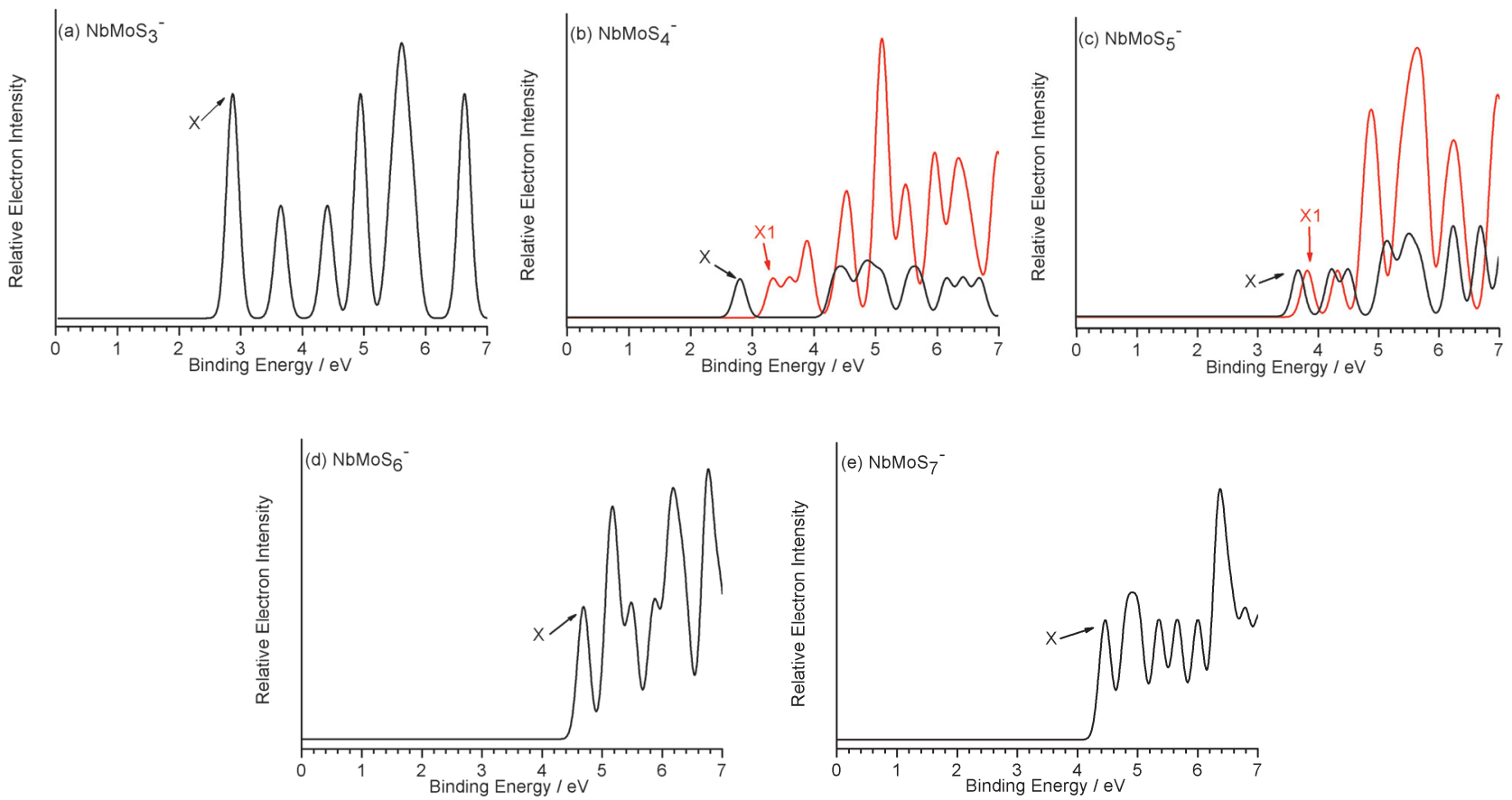

图 6 模拟 $\mathrm{NbMoS}_{n}^{-}(n=3 \sim 7)$ 阴离子基态及其竞争结构的光电子能谱图(黑线: 一重态; 红线: 三重态)

Figure 6 Simulated photoelectron spectra from the ground states and selected low-lying isomers of $\mathrm{NbMoS}_{n}^{-}(n=3 \sim 7)$ clusters at the B3LYP//Basis-II level of theory. (Singlets show in black lines and triplets in red lines) The simulations are done by fitting the distribution of calculated VDEs with unit-area Gaussian functions of $0.1 \mathrm{eV}$ width

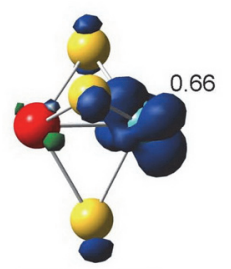

$\mathrm{NbMoS}_{3}\left(C_{s}{ }^{2} \mathrm{~A} "\right)$

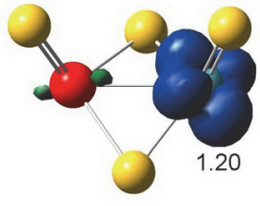

$\mathrm{NbMoS}_{4}\left(C_{s}{ }^{2} \mathrm{~A}^{\prime \prime}\right)$

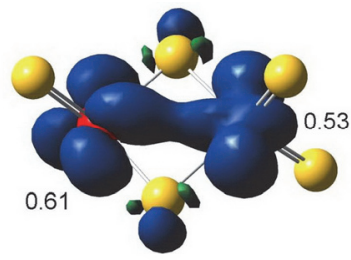

$\operatorname{NbMoS}_{5}\left(C_{s}^{2} \mathrm{~A}^{\prime}\right)$

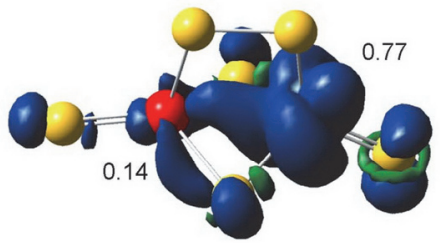

$\mathrm{NbMoS}_{6}\left(C_{s}^{2} \mathrm{~A}^{\prime}\right)$

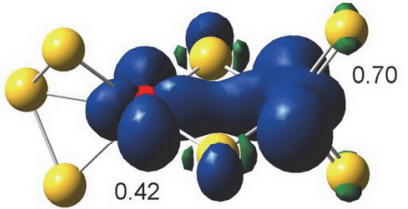

$\mathrm{NbMoS}_{7}\left(C_{s}{ }^{2} \mathrm{~A}^{\prime}\right)$

图 $7 \mathrm{NbMoS}_{n}(n=3 \sim 7)$ 基态构型的电子自旋密度(|e|)示意图(蓝色区域代表单电子的分布, 黑色数值代表自旋密度值)

Figure 7 Electron spin density (in $|\mathrm{e}|)$ for the ground states of $\operatorname{NbMoS}_{n}(n=3 \sim 7)$ clusters

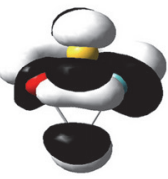

$\left(24 a^{\prime}\right)^{2}$

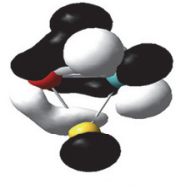

$\left(14 a^{\prime \prime}\right)^{1}$

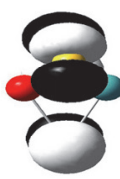

$(13 a ")^{2}$

(a) $\operatorname{NbMoS}_{3}\left(C_{s}{ }^{2} A^{\prime \prime}\right)$

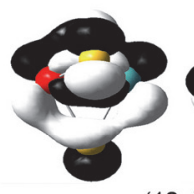

$(12 \mathrm{e})^{4}$

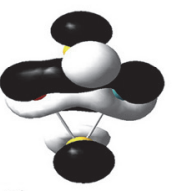

(b) $\mathrm{NbMoS}_{3}{ }^{-}\left(C_{3 v}{ }^{1} \mathrm{~A}_{1}\right)$

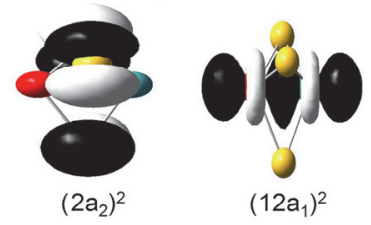

$\left(12 \mathrm{a}_{1}\right)^{2}$

图 $8 \mathrm{NbMoS}_{3}^{-/ 0}$ 基态的价层分子轨道图

Figure 8 Valence molecular orbitals for the ground states of (a) $\mathrm{NbMoS}_{3}$ and (b) $\mathrm{NbMoS}_{3}^{-}$ 


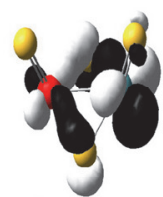

$\left(16 a^{\prime \prime}\right)^{1}$

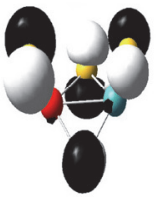

$(15 a ")^{2}$

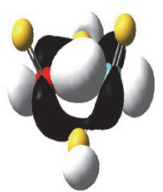

$\left(30 a^{\prime}\right)^{2}$

(a) $\mathrm{NbMoS}_{4}\left(C_{s}{ }^{2} \mathrm{~A}^{\prime \prime}\right)$

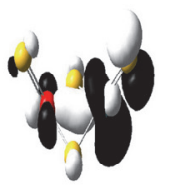

$\left(31 a^{\prime}\right)^{1}$

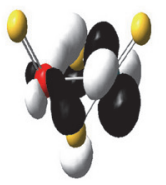

$(16 \mathrm{a}))^{1}$

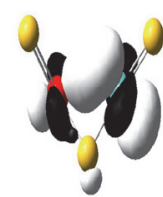

$\left(30 a^{\prime}\right)^{2}$

(b) $\mathrm{NbMoS}_{4}^{-}\left(C_{s}{ }^{3} \mathrm{~A}^{\prime \prime}\right)$

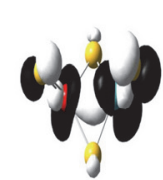

$\left(31 a^{\prime}\right)^{2}$

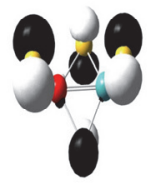

$\left(15 a^{\prime \prime}\right)^{2}$

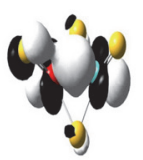

$\left(30 a^{\prime}\right)^{2}$

(c) $\mathrm{NbMoS}_{4}^{-}\left(C_{s}^{1} A^{\prime}\right)$

图 $9 \mathrm{NbMoS}_{4}^{-/ 0}$ 基态及其竞争结构的价层分子轨道图

Figure 9 Valence molecular orbitals for the ground states and selected low-lying isomer of $\mathrm{NbMoS}_{4}$ and $\mathrm{NbMoS}_{4}^{-}$

层 HOMO $\left(31 \mathrm{a}^{\prime}\right)^{2}$ 主要为金属铌和钿上的 $4 \mathrm{~d}$ 轨道成分. 打掉该双占据轨道( $\mathrm{HOMO})$ 上的一个电子会产生谱图 的第一点 VDE 值, 即 $2.81 \mathrm{eV}$ (表 1), 对应谱图的第一 个谱峰(对应图 $6 \mathrm{~b}$ 黑线上 $\mathrm{X}$ 位置). 另外基态阴离子存 在一个能量靠近且构型相近的三重态结构 $\left(C_{s}{ }^{3} \mathrm{~A} "\right)$, 其 价电子组态是 $\cdots\left(29 a^{\prime}\right)^{2}\left(15 a^{\prime \prime}\right)^{2}\left(30 a^{\prime}\right)^{2}\left(16 a^{\prime \prime}\right)^{1}\left(31 a^{\prime}\right)^{1}$ (图 $9 b)$. 其 SOMO $\left(31 \mathrm{a}^{\prime}\right)^{1}$ 轨道主要为金属间 “头碰头”形 成的 $4 \mathrm{~d}-\sigma$ 成键轨道; 次外层的 $\left(16 \mathrm{a}^{\prime \prime}\right)^{1}$ 对应中性的单占 据轨道. 另外我们也模拟了其光电子能谱(对应图 $6 \mathrm{~b}$ 红线上 X1 位置), 对应的第一点 VDE 值为 $3.33 \mathrm{eV}$ (表 $1)$.

\subsection{3 $\mathrm{NbMoS}_{5}$ 和 $\mathrm{NbMoS}_{5}^{-}$}

中性基态 $\mathrm{NbMoS}_{5}\left(C_{s}{ }^{2} \mathrm{~A}^{\prime}\right)$ 的价电子组态为 $\cdots\left(35 \mathrm{a}^{\prime}\right)^{2}$ $\left(16 a^{\prime \prime}\right)^{2}\left(17 a^{\prime \prime}\right)^{2}\left(36 a^{\prime}\right)^{2}\left(37 a^{\prime}\right)^{1}$. 其中 SOMO (37a') 轨道的主 要成分是金属铌钼之间的 $4 \mathrm{~d}$ 轨道; $\left(36 \mathrm{a}^{\prime}\right)^{2}$ 及以下的分子 轨道主要成分为端硫和桥硫的 $3 p$ 轨道成分. 通过自旋 密度图能看到单电子主要位于金属铌和钼上. 阴离子基 态 $\mathrm{NbMoS}_{5}^{-}\left(C_{s}{ }^{1} \mathrm{~A}^{\prime}\right)$ 可认为是在中性 $\mathrm{NbMoS}_{5}$ 基态的 $\operatorname{SOMO}\left(37 \mathrm{a}^{\prime}\right)^{1}$ 添加一个电子而来, 其价电子组态是 $\cdots\left(16 a^{\prime \prime}\right)^{2}\left(17 a^{\prime \prime}\right)^{2}\left(36 a^{\prime}\right)^{2}\left(37 a^{\prime}\right)^{2}$ (图 $10 \mathrm{~b}$ ). 打掉阴离子 $\mathrm{NbMoS}_{5}^{-}$基态 $\mathrm{HOMO}$ 上的一个电子会产生第一个谱峰 (对应图 6c 黑线上 X位置), 对应的 VDE 值是 $3.68 \mathrm{eV}$ (表 1). 另外基态阴离子存在一个能量靠近且构型相近的三 重态结构 $\left(C_{s}{ }^{3} \mathrm{~A}^{\prime \prime}\right)$, 其价电子组态是 $\cdots\left(35 \mathrm{a}^{\prime}\right)^{2}\left(17 \mathrm{a}^{\prime \prime}\right)^{2}$ $\left(36 a^{\prime}\right)^{2}\left(37 a^{\prime}\right)^{1}\left(38 a^{\prime}\right)^{1}$ (图 10c). 其 SOMO (38a') 轨道主要为 金属铌和钿上两种类型的 $4 \mathrm{~d}$ 轨道形成较弱的 $4 \mathrm{~d}-\sigma$ 成键; 次外层的 $\left(37 \mathrm{a}^{\prime}\right)^{1}$ 单占据轨道金属间形成非键. 与此同时 我们也模拟了三重态异构体 $\left(C_{s}{ }^{3} \mathrm{~A}^{\prime}\right)$ 的光电子能谱(图 $6 \mathrm{c}$ 红线上 X1 位置), 其第一点 VDE 计算值为 $3.82 \mathrm{eV}$ (表 $1)$.

$$
\begin{aligned}
& \text { 3.2.4 } \mathrm{NbMoS}_{6} \text { 和 } \mathrm{NbMoS}_{6}^{-} \\
& \text {中性基态 } \mathrm{NbMoS}_{6}\left(C_{s}{ }^{2} \mathrm{~A}^{\prime}\right) \text { 的价电子组态是 }
\end{aligned}
$$

$\cdots\left(17 a^{\prime \prime}\right)^{2}\left(42 a^{\prime}\right)^{2}\left(18 a^{\prime \prime}\right)^{2}\left(19 a^{\prime \prime}\right)^{2}\left(43 a^{\prime}\right)^{1}$. 其中 SOMO $\left(43 a^{\prime}\right)^{1}$ 主要成分是由金属钿和铌上 $4 \mathrm{~d}$ 轨道以 “头碰头” 形式 形成的 $\sigma$ 成键轨道; 次外层轨道 $\left(19 \mathrm{a}^{\prime \prime}\right)^{2}$ 主要为桥位硫的 $\mathrm{S}_{2}$ 基团、桥硫和桥端硫上含明显的 $3 p$ 轨道; $\left.(18 \mathrm{a})^{2}\right)^{2}$ 主要 是位于 $\mathrm{S}_{2}^{2-}$ 基团上的两个硫存在强烈的 $\pi^{*}$ 反键轨道. 自 旋密度图(图 7)能看到单电子主要位于金属钼上. 阴离 子基态 $\mathrm{NbMoS}_{6}^{-}\left(C_{s}{ }^{1} \mathrm{~A}^{\prime}\right)$ 的价电子组态是 $\cdots\left(18 \mathrm{a}^{\prime \prime}\right)^{2}$ $\left(42 a^{\prime}\right)^{2}\left(19 a^{\prime \prime}\right)^{2}\left(43 a^{\prime}\right)^{2}$. HOMO $\left(43 a^{\prime}\right)^{2}$ 及以下分子轨道 $3 p$ 成 分较为明显, 即端硫和桥硫上的 $3 p$ 轨道. 打掉基态阴离 子 $\mathrm{NbMoS}_{6}^{-} \mathrm{HOMO}$ 轨道上的一个电子会产生第一个谱 峰(对应图 $6 \mathrm{~d}$ 黑线上 X 位置), 对应的 VDE 值是 $4.69 \mathrm{eV}$ (表 1).
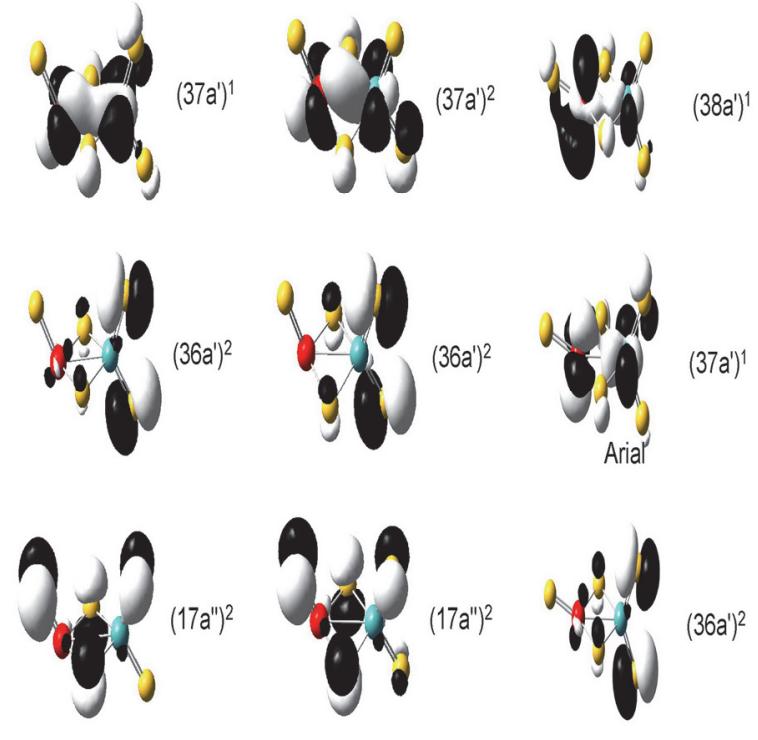

(a) $\mathrm{NbMOS}_{5}\left(C_{\mathrm{s}}{ }^{2} \mathrm{~A}^{\prime}\right)$

(b) $\operatorname{NbMoS}_{5}^{-}\left(C_{S}^{1} A^{\prime}\right)$

图 $10 \mathrm{NbMoS}_{5}^{-10}$ 基态及其竞争结构的价层分子轨道图

Figure 10 Valence molecular orbitals for the ground states and selected low-lying isomer of $\mathrm{NbMoS}_{5}$ and $\mathrm{NbMoS}_{5}^{-}$ 

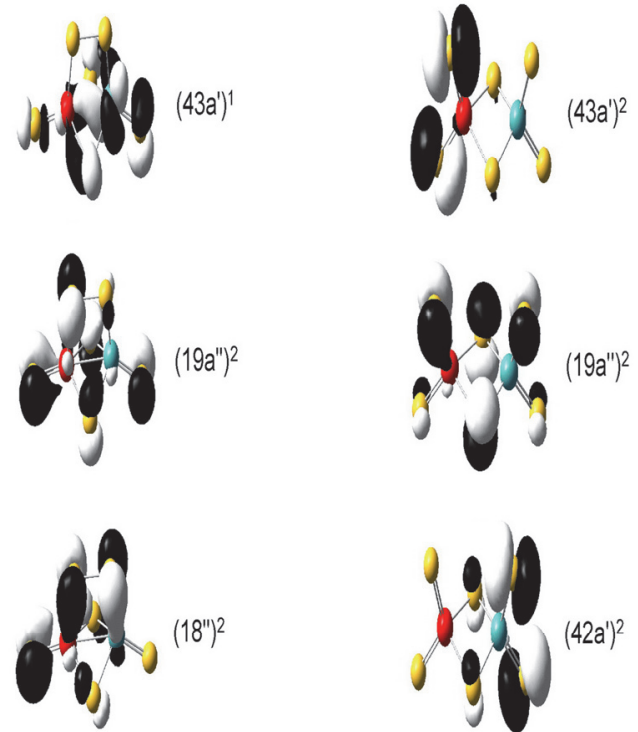

(a) $\mathrm{NbMOS}_{6}\left(\mathrm{C}_{s}^{2} A^{\prime}\right)$

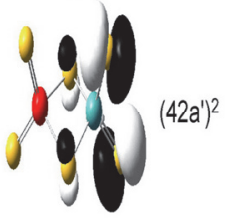

(b) $\operatorname{NbMoS}_{6}^{-}\left(C_{s}^{1} A^{\prime}\right)$

图 11 基态 $\mathrm{NbMoS}_{6}^{-/ 0}$ 的价层分子轨道图

Figure 11 Valence molecular orbitals for the ground states of (a) $\mathrm{NbMoS}_{6}$ and (b) $\mathrm{NbMoS}_{6}^{-}$

\subsection{5 $\mathrm{NbMoS}_{7}$ 和 $\mathrm{NbMoS}_{7}^{-}$}

中性基态 $\mathrm{NbMoS}_{7}\left(C_{s}{ }^{2} \mathrm{~A}^{\prime}\right)$ 的价电子组态是 $\cdots\left(43 a^{\prime}\right)^{2}\left(44 a^{\prime}\right)^{2}\left(24 a^{\prime \prime}\right)^{2}\left(25 a^{\prime \prime}\right)^{2}\left(45 a^{\prime}\right)^{1}$. 其 SOMO 轨道 $\left(45 \mathrm{a}^{\prime}\right)^{1}$ 主要成分为金属铌和钿间的 $4 \mathrm{~d}-\sigma$ 成键轨道. 自旋 密度图(图 7)看到单电子主要位于金属铌和钼上. 基态 阴离子 $\mathrm{NbMoS}_{7}^{-}\left(C_{1}{ }^{1} \mathrm{~A}\right)$ 的价电子组态是 $\cdots(67 \mathrm{a})^{2}(68 \mathrm{a})^{2}-$ $(69 a)^{2}(70 a)^{2}$. HOMO 轨道(70a $)^{2}$ 主要成分为 $S_{2}^{2-}$ 基团上 两个 $3 \mathrm{p}$ 轨道形成的 $\pi^{*}$ 反键轨道; 而次外层的 $(69 \mathrm{a})^{2}$ 轨道 主要为端硫和桥硫上的 $3 \mathrm{p}$ 轨道成分. 打掉基态阴离子 $\mathrm{NbMoS}_{7}^{-}$的 $\mathrm{HOMO}$ 轨道上的一个电子会产生第一个谱 峰(对应图 6e 黑线上 X 位置), 对应的 VDE 值是 $4.46 \mathrm{eV}$ (表 1).

\section{3 $\mathrm{NbMoS}_{n}^{-}(n=3 \sim 7)$ 的 $\mathrm{VDE}_{1 \mathrm{st}}$ 与 ADE 随硫含量的 变化趋势}

图 13 是 $\mathrm{NbMoS}_{n}^{-}(n=3 \sim 7)$ 基态的 $\mathrm{VDE}_{1 \mathrm{st}}$ 与 $\mathrm{ADE}$ 随硫含量变化而变化的趋势图, 我们能看到明显的两个 趋势: 其一, $\mathrm{VDE}_{1 \mathrm{st}}$ 与 $\mathrm{ADE}$ 随硫含量增加的变化趋势是 基本一致的; 其二, 随着硫含量的增加对应的 $\mathrm{VDE}_{1 \mathrm{st}}$ 与 $\mathrm{ADE}$ 呈现先增后降的趋势, 即当 $n=6$ 时 $\mathrm{VDE}_{1 \mathrm{st}}$ 与 $\mathrm{ADE}$ 达到了最大值 $\left(\mathrm{ADE}=4.27 \mathrm{eV} ; \mathrm{VDE}_{1 \mathrm{st}}=4.69 \mathrm{eV}\right)$, 而从 $\mathrm{NbMoS}_{6}^{-}$到 $\mathrm{NbMoS}_{7}^{-}$, 对应的 $\mathrm{VDE}_{1 \mathrm{st}}$ 与 $\mathrm{ADE}$ 值开始降 低(即先增后降). 出现这样趋势的原因可结合前面的各 体系价层分子轨道(图 8 12) 分析得知: 当 $n \leqslant 6$ 时随着 硫含量 $n$ 增加, 金属 $\mathrm{Mo}\left(4 \mathrm{~d}^{5} 5 \mathrm{~s}^{1}\right)$ 和 $\mathrm{Nb}\left(4 \mathrm{~d}^{4} 5 \mathrm{~s}^{1}\right)$ 原子上的 价电子因要与硫原子成键而逐渐减少, 金属原子的氧化 态逐渐增大, 相应地体系第一点 $\operatorname{VDE}\left(\mathrm{VDE}_{1 \mathrm{st}}\right)$ 和 $\mathrm{ADE}$ 值 均呈现逐渐增大的趋势; 在 $n=6$ 时铌和钿达到
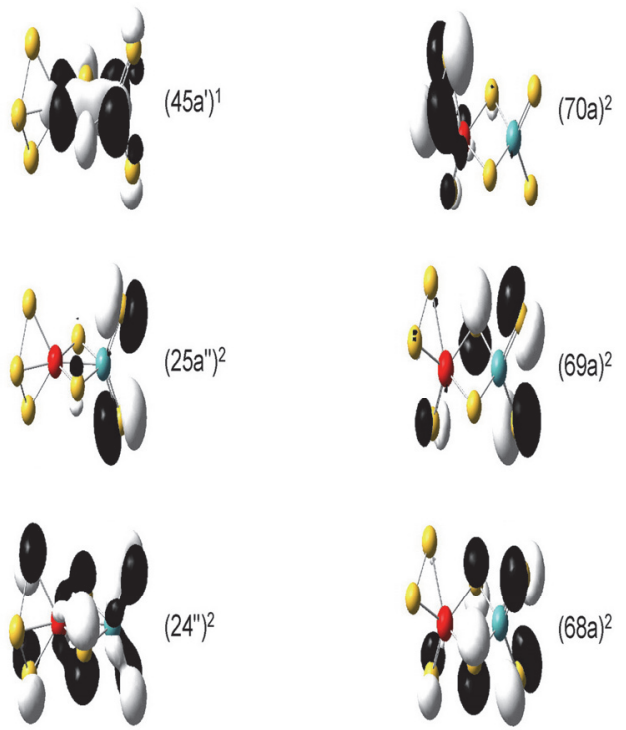

(a) $\operatorname{NbMoS}_{7}\left(C_{S}{ }^{2} A^{\prime}\right)$

(b) $\operatorname{NbMoS}_{7}^{-}\left(C_{1}{ }^{1} A\right)$

图 12 基态 $\mathrm{NbMoS}_{7}^{-/ 0}$ 的价层分子轨道图

Figure 12 Valence molecular orbitals for the ground states of (a) $\mathrm{NbMoS}_{7}$ and (b) $\mathrm{NbMoS}_{7}^{-}$

它们的最高氧化态 +5 和 +6 , 此时 $\mathrm{VDE}_{1 \mathrm{st}}$ 和 $\mathrm{ADE}$ 值也 达到最大值. 在此过程中较为特别的是, 从 $\mathrm{NbMoS}_{3}^{-}$到 $\mathrm{NbMoS}_{4}^{-}$的 $\mathrm{VDE}_{1 \mathrm{st}}$ 和 $\mathrm{ADE}$ 值有略微下降，其原因可以 理解为体系由三桥结构变为双桥结构, 这时金属-金属 间键长增长 $(2.062 \AA \rightarrow 2.487 \AA)$, 金属一金属间成键变弱, 那么打掉该成键轨道上的 $4 \mathrm{~d}$ 电子所需能量减少, 对应 的 $\mathrm{VDE}_{1 \mathrm{st}}$ 和 $\mathrm{ADE}$ 值也相应变小; 当 $n=7$ 时, $\mathrm{VDE}_{1 \mathrm{st}}$ 和 $\mathrm{ADE}$ 值的下降可理解为相应的电子是从 $\mathrm{NbMoS}_{7}^{-}$上的 $\mathrm{S}_{2}$ 基团 $\pi^{*}$ 反键轨道上被打掉, 从反键轨道上打掉电子 所需能量较少.

\section{4 $\mathrm{NbMoS}_{n}^{-/ 0}(n=3 \sim 7)$ 的结构演化和顺序硫化}

$\mathrm{NbMoS}_{n}^{-/ 0}(n=3 \sim 7)$ 的基态构型如图 14 所示. 对 于中性 $\mathrm{NbMoS}_{n}(n=3 \sim 7)$ 而言, 可以认为 $\mathrm{NbMoS}_{3}$ 的基 态构型是在 $\mathrm{NbMoS}_{2}$ 四元环的基础上先增加一个桥硫原 子得到; 而 $\mathrm{NbMoS}_{4}$ 基态构型是在 $\mathrm{NbMoS}_{2}$ 四元环基础 上增加两个端硫原子, 且这两个端硫原子分别加到两种 金属原子上得到; 在 $\mathrm{NbMoS}_{4}$ 基态构型基础上，再继续 增加一个硫原子, 由于钼原子比铌原子剩余的 $4 \mathrm{~d}$ 价电 子数更多, 所以新添的硫原子以端硫形式加到金属钿原 子上, 得到 $\mathrm{NbMoS}_{5}$ 基态构型; 继续增加一个硫原子, 新添的硫原子加到还剩余一个单电子的铌原子上形成 硫自由基, 硫自由基再与钿上的一个端硫原子形成桥连 的 $\mathrm{S}_{2}$ 基团，得到 $\mathrm{NbMoS}_{6}$ 基态构型; 而 $\mathrm{NbMoS}_{7}$ 可以认 为是在 $\mathrm{NbMoS}_{5}$ 结构基础上, 于铌端新增一个 $\mathrm{S}_{2}$ 基团, 形成 $\mathrm{S}_{2}^{-}$自由基, 然后该 $\mathrm{S}_{2}^{-}$自由基与铌上的端硫进一步 形成 $\mathrm{S}_{3}$ 基团. 


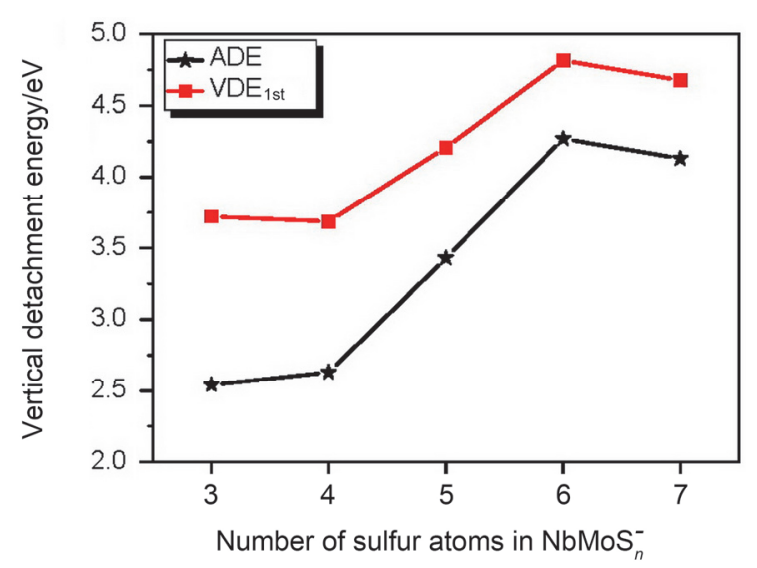

图 13 基态阴离子 $\mathrm{NbMoS}_{n}^{-}(n=3 \sim 7) \mathrm{VDE}_{1 \mathrm{st}}$ 与 $\mathrm{ADE}$ 随硫含量的变 化趋势图

Figure 13 The calculated $\mathrm{VDE}_{1 \mathrm{st}}$ and $\mathrm{ADE}$ of $\operatorname{NbMoS}_{n}^{-}(n=3 \sim 7)$ as a function of $\mathrm{S}$ content $(n)$

相应的 $\mathrm{NbMoS}_{n}^{-}(n=3 \sim 7)$ 阴离子基态也有类似的 结构演化规律, 在硫含量 $n<6$ 时, 阴离子基态结构与中 性基态构型基本类似. 当硫含量 $n=5$ 时, 由于铌原子上 仍富余两个价电子, 新增的硫原子加到铌原子上形成端 硫, 得到 $\mathrm{NbMoS}_{6}^{-}$的阴离子基态结构; 对于富硫类型 $\mathrm{NbMoS}_{7}^{-}$阴离子, 由于铌和钿上已无多余的价电子, 所 以新增的硫原子将加到铌的一个端硫原子上, 形成 $\mathrm{S}_{2}$ 基团.

\section{5 $\mathrm{NbMoS}_{n}^{-/ 0}(n=3 \sim 7)$ 的相对稳定性}

为了进一步比较该系列团簇在硫含量改变情况下 的相对稳定性, 我们分别计算了各基态结构的平均结合 能 $\left(E_{\mathrm{b}}\right)$ 与能量二阶差分 $\left(\Delta_{2} E\right)$ 值. 我们所采用的计算公式 如下 ${ }^{[82,83]}$ :

$$
\begin{aligned}
& E_{\mathrm{b}}\left(\mathrm{NbMoS}_{n}\right)=[ n E_{\mathrm{k}}(\mathrm{S})+E_{\mathrm{k}}(\mathrm{Nb})+E_{\mathrm{k}}(\mathrm{Mo})- \\
&\left.E_{\mathrm{k}}\left(\mathrm{NbMoS}_{n}\right)\right] /(n+2) \\
& E_{\mathrm{b}}\left(\mathrm{NbMoS}_{n}^{-}\right)=\left[(n-1) E_{\mathrm{k}}(\mathrm{S})+E_{\mathrm{k}}\left(\mathrm{S}^{-}\right)+E_{\mathrm{k}}(\mathrm{Nb})+\right. \\
&\left.E_{\mathrm{k}}(\mathrm{Mo})-E_{\mathrm{k}}\left(\mathrm{NbMoS}_{n}\right)\right] /(n+2)
\end{aligned}
$$

$$
\begin{aligned}
& \Delta_{2} E\left(\mathrm{NbMoS}_{n}^{-/ 0}\right)= E_{\mathrm{k}}\left(\mathrm{NbMoS}_{n+1}^{-/ 0}\right)+ \\
& E_{\mathrm{k}}\left(\mathrm{NbMoS}_{n-1}^{-1}\right)-2 E_{\mathrm{k}}\left(\mathrm{NbMoS}_{n}^{-/ 0}\right)
\end{aligned}
$$

图 15 展示了 $\mathrm{NbMoS}_{n}^{-/ 0}(n=3 \sim 7)$ 团簇平均结合能 $\left(E_{\mathrm{b}}\right)$ 和能量二阶差分 $\left(\Delta_{2} E\right)$ 值随硫含量 $n$ 的变化关系曲 线. 从图中可以看出, 不管是平均结合能 $\left(E_{\mathrm{b}}\right)$ 的关系曲 线, 还是能量二阶差分 $\left(\triangle_{2} E\right)$ 的关系曲线, 其趋势基本 一致. 在中性 $\mathrm{NbMoS}_{n}$ 团簇中, 以 $\mathrm{NbMoS}_{5}$ 的 $E_{\mathrm{b}}$ 和 $\Delta_{2} E$ 值最大, 表明它与其他团簇相比具有更高的稳定性; 而 阴离子 $\mathrm{NbMoS}_{n}^{-}$簇中, $\mathrm{NbMoS}_{6}^{-}$的 $E_{\mathrm{b}}$ 和 $\Delta_{2} E$ 值最大, 说 明它比其它相邻的团簇更加稳定.

\section{$3.6 \mathrm{H}_{2}$ 在 $\mathrm{NbMoS}_{n}^{-/ 0}(n=3 \sim 7)$ 簇合物上的还原反应}

Afanasiev 等 ${ }^{[84 ~ 86]}$ 研究发现 $\mathrm{MoS}_{2}$ 催化剂边缘位上 的 $S_{2}^{2-}$ 对催化加氢脱硫(HDS)反应活性有着重要的影响, 如 $\mathrm{MoS}_{2}$ 催化剂常在高温 $\mathrm{H}_{2} / \mathrm{H}_{2} \mathrm{~S}$ 气氛中使用, $\mathrm{S}_{2}^{2-}$ 基团

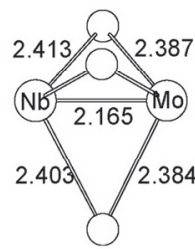

$\operatorname{NbMoS}_{3}\left(C_{s}{ }^{2} A^{\prime \prime}\right)$

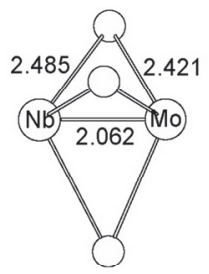

$\mathrm{NbMoS}_{3}{ }^{-}\left(C_{3 v}{ }^{1} \mathrm{~A}_{1}\right)$

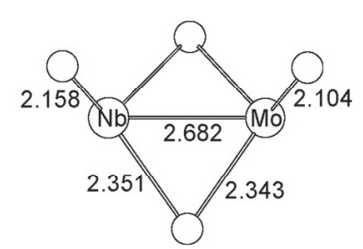

$\mathrm{NbMoS}_{4}\left(C_{s}{ }^{2} A^{\prime \prime}\right)$

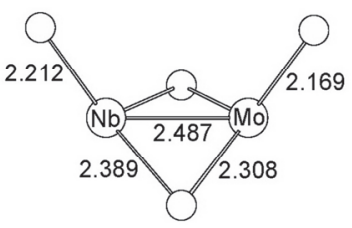

$\mathrm{NbMoS}_{4}^{-}\left(C_{s}{ }^{1} A^{\prime}\right)$

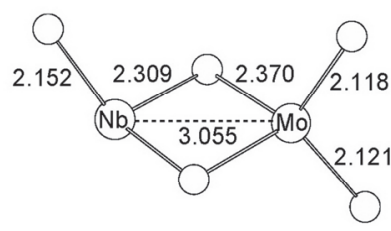

$\operatorname{NbMoS}_{5}\left(C_{s}{ }^{2} A^{\prime}\right)$

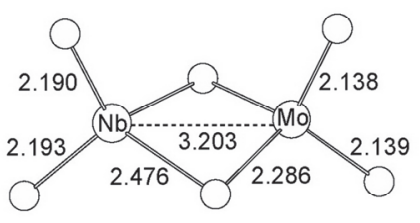

$\operatorname{NbMoS}_{6}^{-}\left(C_{s}{ }^{1} A^{\prime}\right)$

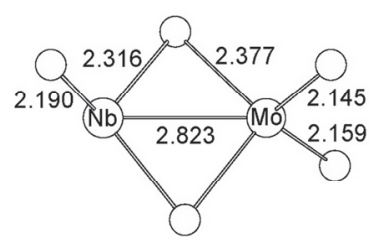

$\operatorname{NbMoS}_{5}^{-}\left(C_{s}^{1} A^{\prime}\right)$

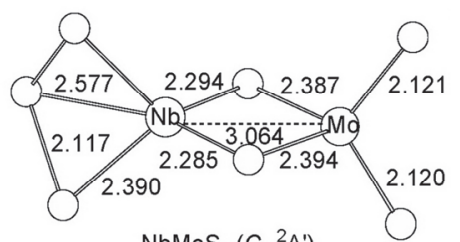

$\operatorname{NbMoS}_{7}\left(C_{s}{ }^{2} A^{\prime}\right)$

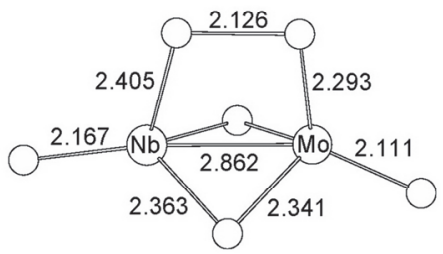

$\mathrm{NbMoS}_{6}\left(C_{s}{ }^{2} A^{\prime}\right)$

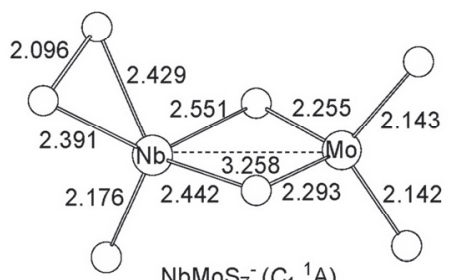

图 14 在 B3LYP/Basis-II 水平下得到的基态 $\mathrm{NbMoS}_{n}^{-/ 0}(n=3 \sim 7)$ 构型

Figure 14 Optimized ground states for $\operatorname{NbMoS}_{n}^{-/ 0}(n=3 \sim 7)$ clusters at the B3LYP/Basis-II level of theory 

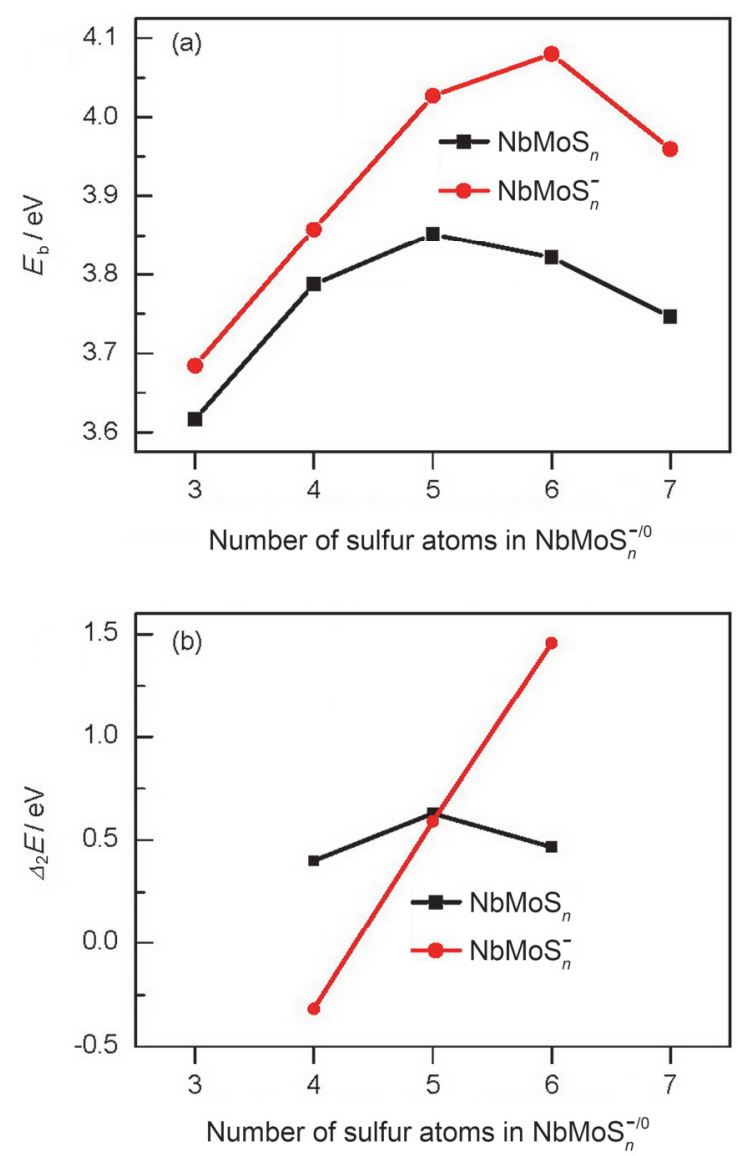

图 $15 \mathrm{NbMoS}_{n}^{-/ 0}(n=3 \sim 7)$ 团簇的平均结合能 $E_{\mathrm{b}}$ 与能量二阶差分 $\Delta_{2} E$ 随硫含量的变化趋势图

Figure 15 The averaged binding energy $\left(E_{\mathrm{b}}\right)$ and second-order energy difference $\left(\Delta_{2} E\right)$ of $\operatorname{NbMoS}_{n}^{-/ 0}(n=3 \sim 7)$ as a function of $\mathrm{S}$ content $(n)$

表 2 在 B3LYP/Basis-II 水平下得到的反应(4)吉布斯自由能变化值 $\Delta G$

Table 2 Calculated free energy differences $(\Delta G)$ for the proposed reaction (4) at the B3LYP/Basis-II level of theory

\begin{tabular}{ccc}
\hline$n \rightarrow n-1$ & $\Delta G_{\mathrm{nu}}^{a, b}$ & $\Delta G_{\mathrm{an}}^{a, b}$ \\
\hline $4 \rightarrow 3$ & 34.92 & 37.07 \\
$5 \rightarrow 4$ & 25.09 & 43.44 \\
$6 \rightarrow 5$ & 9.90 & 30.96 \\
$7 \rightarrow 6$ & 0.77 & -3.43 \\
\hline
\end{tabular}

$\overline{{ }^{a} \Delta G_{\text {nu }} \text { and } \Delta G_{\text {an }} \text { mean } \Delta G \text { of the neutral and anionic clusters, respectively. }{ }^{b} \text { All }}$ energies are in $\mathrm{kcal} \cdot \mathrm{mol}^{-1}$.

能促进 $\mathrm{H}_{2}$ 解离成活性氢原子 ${ }^{[87]}$. 此外, 铌硫化物也被 报道在 HDS 等反应中表现出了优异的催化性能 ${ }^{[88]}$. 因 此铌钼掺杂的双金属硫化物在 HDS 反应中能否引起催 化性能的提高成为我们感兴趣的内容. 为此, 我们设计 了如下的反应(反应 4), 来评估掺杂后铌钼硫化物团簇 与 $\mathrm{H}_{2}$ 反应, 最终以 $\mathrm{H}_{2} \mathrm{~S}$ 形式脱去一个 $\mathrm{S}$ 原子的反应驱 动力 $(-\Delta G)$ 大小, $\Delta G$ 的大小通过方程 5 进行计算得到:

$$
\mathrm{NbMoS}_{n}^{-/ 0}+\mathrm{H}_{2} \rightleftharpoons \mathrm{NbMoS}_{n-1}^{-/ 0}+\mathrm{H}_{2} \mathrm{~S}
$$

$$
\begin{aligned}
\Delta G= & G\left(\mathrm{NbMoS}_{n-1}^{-/ 0}\right)+G\left(\mathrm{H}_{2} \mathrm{~S}\right)- \\
& G\left(\mathrm{NbMoS}_{n}^{-/ 0}\right)-G\left(\mathrm{H}_{2}\right)
\end{aligned}
$$

反应 4 的 $\Delta G$ 结果列于表 2 中. 根据计算结果发现, 对于中性 $\operatorname{NbMoS}_{n}(n=3 \sim 7)$, 其与 $\mathrm{H}_{2}$ 反应的 $\Delta G$ 值随硫 含量 $n$ 的增加而降低, 且 $\Delta G$ 值均是正值, 这说明中性体 系与 $\mathrm{H}_{2}$ 的反应正向非自发. 对于阴离子体系, 发现 $\Delta G$ 值随硫含量 $n$ 的增加出现先增加后减小的趋势, 当 $n<7$ 时 $\Delta G$ 值均为正值, 当 $n=7$ 时 $\Delta G$ 值出现负值, 出现负值 说明此时反应正向自发. 这说明催化剂的预硫化, 使催 化剂表面形成端连的 $\mathrm{S}_{2}^{2-}$ 基团，该基团对形成具有催化 活性的配位不饱和位(CUS) 有重要作用. 且形成的 $\mathrm{S}_{2}^{2-}$ 基团倾向形成于铌原子上，也说明硫化铌更容易在催化 剂表面形成 $\mathrm{S}_{2}^{2-}$ 基团并被活化为 CUS 活性位. 我们预计 在硫化钼催化剂中掺杂一定比例的铌原子有利于提高 $\mathrm{MoS}_{2}$ 催化剂的加氢脱硫活性.

\section{7 中性基态 $\operatorname{NbMoS}_{n}(n=3 \sim 7)$ 的路易斯酸性}

路易斯酸性强弱, 可以在一定程度上说明其亲电能 力的强弱. 关于团簇路易斯酸性的大小可通过计算其氟 亲和能(FAs)得到 ${ }^{[89,90]}$, 而 FA 值被定义为反应 6 焓变的 负值(方程 7).

$$
\begin{aligned}
& \mathrm{A}+\mathrm{F}^{-}=\mathrm{AF}^{-} \\
& \mathrm{FA}=-\left[\mathrm{H}\left(\mathrm{AF}^{-}\right)-\mathrm{H}(\mathrm{A})-\mathrm{H}\left(\mathrm{F}^{-}\right)\right] /(\mathrm{eV})
\end{aligned}
$$

通过上面关于路易斯酸性的定义, 我们计算得到了 $\mathrm{NbMoS}_{n}(n=3 \sim 7)$ 的 FA 值(表 3), 通过比较我们发现, FA 值随硫含量的增加出现先增加后减小的趋势. 从 $n=$ 3 到 $n=5$, FA 值先增加, 在 $\mathrm{NbMoS}_{5}$ 时 $\mathrm{FA}$ 值达到最大 值 $5.69 \mathrm{eV}$. 这是由于随硫含量增加, 金属位上的电子逐 渐转移到硫原子上参与成键, 金属原子的氧化态逐渐增 大. 当硫含量继续增大, $\mathrm{NbMoS}_{6}$ 和 $\mathrm{NbMoS}_{7}$ 中开始分别 出现 $\mathrm{S}_{2}^{2-}$ 基团和 $\mathrm{S}_{3}^{2-}$ 基团. $\mathrm{F}^{-}$和 $\mathrm{S}_{2}^{2-}$ 基团均带负电荷，因 此它们间存在较强的静电排斥, 且 $\mathrm{NbMoS}_{6}$ 和 $\mathrm{NbMoS}_{7}$ 的空间位阻较大, 因而 FA 值从 $n=6$ 开始下降. 此外, 对 $\mathrm{NbMoS}_{n} \mathrm{~F}^{-}(n=3 \sim 7)$ 最稳构型的搜索, 我们是在 $\mathrm{NbMoS}_{n}(n=3 \sim 7)$ 最稳定构型的基础上考虑氟阴离子 的不同吸附位置, 包括在 $\mathrm{Nb}$ 位、Mo 位、端硫位以及桥 硫位上的情况. 最终优化得到的结果见于附录图 S12. 我们发现 $\mathrm{NbMoS}_{n} \mathrm{~F}^{-}(n=3 \sim 7)$ 的最稳构型中, $\mathrm{F}^{-}$倾向 直接与金属铌原子成键，这也说明团簇上的铌原子位有 较强的亲电能力. 上述数据能为未来改进该类材料的催 化活性提供一定的理论依据.

表 3 方程(7)的氟亲和能 FA

Table 3 Equation (7) for the values of fluorine affinity (FA)

\begin{tabular}{cc||cc}
\hline Cluster & $\mathrm{FA}^{a}$ & Cluster & $\mathrm{FA}^{a}$ \\
\hline $\mathrm{NbMoS}_{3}$ & 4.68 & $\mathrm{NbMoS}_{6}$ & 4.37 \\
$\mathrm{NbMoS}_{4}$ & 5.18 & $\mathrm{NbMoS}_{7}$ & 3.77 \\
$\mathrm{NbMoS}_{5}$ & 5.69 & & \\
\hline
\end{tabular}

${ }^{a}$ All energies are in $\mathrm{eV}$. 


\section{4 结论}

本论文采用密度泛函(DFT)与耦合簇 $[\mathrm{CCSD}(\mathrm{T})]$ 方 法对一系列掺杂类型的二核 $\mathrm{NbMoS}_{n}^{-/ 0}(n=3 \sim 7)$ 团簇 体系进行广泛的势能面搜索, 寻找体系最稳定的几何构 型, 并对其在几何、电子结构与化学成键等方面所表现 出来的性质规律进行研究. 在确定 $\mathrm{NbMoS}_{n}^{-}$阴离子基 态结构的基础上, 我们采用广义 Koopmans 定理计算出 电子垂直逸出能(VDE), 并模拟了各阴离子的光电子能 谱, 结合对分子轨道的分析进一步讨论该铌钼硫掺杂团 族体系在顺序硫化过程中的演变规律. 结果表明: $\mathrm{NbMoS}_{n}^{-/ 0}(n=3 \sim 7)$ 基态可视为在 $\mathrm{NbMoS}_{2}$ 四元环基础 上逐渐加硫演变而来, 各种硫配体出现的先后顺序如 下: 在金属 $\mathrm{Nb}$ 和金属 $\mathrm{Mo}$ 分别达到其最高氧化态 +5 和 +6 之前, $\mathrm{S}$ 以桥位或端位形式出现, 当 $\mathrm{Nb}$ 和 $\mathrm{Mo}$ 达到 最高氧化态后, $\mathrm{Nb}$ 和 $\mathrm{Mo}$ 原子上没有多余的价电子用来 成键, 因此开始出现桥连或端连的 $\mathrm{S}_{2}$ 基团, 硫含量继续 增大, 最后出现端连的 $\mathrm{S}_{3}$ 基团; 我们还对 $\mathrm{NbMoS}_{n}^{-/ 0}$ 才 簇体系与 $\mathrm{H}_{2}$ 反应的驱动力进行计算, 结果发现 $\mathrm{S}_{2}^{2-}$ 基 团对形成配位不饱和位(CUS)有重要作用. 因而硫化钼 中掺杂适量的 $\mathrm{Nb}$ 以及在催化剂制备时充分预硫化处理, 将对 $\mathrm{S}_{2}^{2-}$ 基团的形成有利, 上述措施有可能提高催化剂 CUS 活性位的浓度, 进而提高其催化活性. 此外, 具有 配位不饱和位的 $\mathrm{NbMoS}_{5}$ 具有最大的路易斯酸性, 有较 好的亲电能力. 上述结果为探索新的掺杂类型过渡金属 硫化物催化剂提供了一定的理论依据和启示.

\section{References}

[1] Shi, J. P.; Ma, D. L.; Zhang, Y. F.; Liu, Z. F. Acta Chim. Sinica 2015, 73, 877. (史建平, 马冬林, 张艳锋, 刘忠范, 化学学报, 2015, 73, 877.)

[2] Transition Metal Sulfur Chemistry: Biological and Industrial Significance, Eds.: Stiefel, E. I.; Matsumoto, K., American Chemical Society, Washington, 1996.

[3] Lee, S. C.; Li, J.; Mitchell, J. C.; Holm, R. H. Inorg. Chem. 1992, $31,4333$.

[4] Nasretdinova, V.; Zaitsev-Zotov, S. Physica B 2012, 407, 1874.

[5] Wang, Q.; Zhao, J.; Wang, X. F. J. Phys. Chem. A 2015, 119, 2244.

[6] Pettarin, V.; Churruca, M. J.; Felhos, D.; Karger-Kocsis, J.; Frontini, P. M. Wear 2010, 269, 31.

[7] Basharina, K. Y.; Terekhin, D. V.; Kuz'mina, G. N.; Bordubanova, A. E.; Ezhov, G. A.; Parenago, O. P. Petrol. Chem. 2009, 49, 339.

[8] Chhowalla, M.; Amaratunga, G. A. J. Nature (London) 2000, 407, 164.

[9] Ye, L. N.; Wu, C. Z.; Guo, W.; Xie, Y. Chem. Commun. 2006, 45, 4738.

[10] Walter, M. G.; Warren, E. L.; McKone, J. R.; Boettcher, S. W.; Mi, Q.; Santori, E. A.; Lewis, N. S. Chem. Rev. 2010, 110, 6446.

[11] Jaramillo, T. F.; Jørgensen, K. P.; Bonde, J.; Nielsen, J. H.; Horch, S.; Chorkendorff, I. Science 2007, 317, 100.

[12] Tian, Y.; He, Y.; Shang, J.; Zhu, Y. F. Acta Chim. Sinica 2004, 62, 1807. (田野, 何俣, 尚静, 朱永法, 化学学报, 2004, 62, 1807.)

[13] Xing, L.; Jiao, L. Y. Acta Phys.-Chim. Sin. 2016, 32, 2133. (邢垒, 焦丽颖, 物理化学学报, 2016, 32, 2133.)

[14] Raybaud, P.; Hafner, J.; Kresse, G.; Kasztelan, S.; Toulhoat, H. J. Catal. 2000, 189, 129.

[15] Toulhoat, H.; Raybaud, P.; Kasztelan, S.; Kresse, G.; Hafner, J. Catal. Today 1999, 50, 629.

[16] Jaramillo, T. F. Nature Chem. 2014, 6, 248.

[17] Jaramillo, T. F.; Jørgensen, K. P.; Bonde, J.; Nielsen, J. H.; Horch,
S.; Chorkendorff, I. Science 2007, 317, 100.

[18] Guo, X.; Tong, X.; Wang, Y.; Chen, C.; Jin, G.; Guo, X. Y. J. Mater. Chem. A 2013, 1, 4657.

[19] Liao, L.; Zhu, J.; Bian, X.; Zhu, L.; Scanlon, M. D.; Girault, H. H.; Liu, B. Adv. Funct. Mater. 2013, 23, 5326.

[20] Kockerling, M.; Johrendt, D.; Finckh, E. W. J. Am. Chem. Soc. 1998 , 120,12297

[21] Hernandez-Molina, R.; Gili, P.; Sokolov, M. N.; Safont, V. S. Inorg. Chim. Acta 2011, 376, 10.

[22] Liao, Y. H.; Park, K. S.; Singh, P.; Li, W.; Goodenough, J. B. J. Power Sources 2014, 245, 27.

[23] Divigalpitiya, W. M. R.; Frindt, R. F.; Morrison, S. R. J. Phys. D: Appl. Phys. 1990, 23, 966.

[24] Oviedo-Roa, R.; Martinez-Magadan, J. M.; Illas, F. J. Phys. Chem. B 2006, 110,7951 .

[25] Lewis, D. A.; Kenney, C. N. Trans. Inst. Chem. Eng. 1981, 59, 186.

[26] Aray, Y.; Zambrano, D.; Cornejo, M. H.; Ludeña, E. V.; Iza, P.; Vidal, A. B.; Coll, D. S.; Jimenez, D. M.; Henriquez, F.; Paredes, C. J. Phys. Chem. C 2014, 118, 27823.

[27] Allali, N.; Marie, A. M.; Danot, M.; Geantet, C.; Breysse, M. J. Catal. 1995, 156, 279

[28] Geantet, C.; Afonso, J.; Breysse, M.; Danot, M. Catal. Today 1996 $28,23$.

[29] Allali, N.; Prouzet, E.; Michalowicz, A.; Gaborit, V.; Nadiri, A.; Danot, M. Appl. Catal. A-GEN. 1997, 159, 333.

[30] Cattenot, M.; Portefaix, J. L.; Afonso, J.; Breysse, M.; Lacroix, M.; Perot, G. J. Catal. 1998, 173, 366.

[31] Danot, M.; Afonso, J.; Portefaix, J. L.; Breysse, M.; Courieres, T. D. Catal. Today 1991, 10, 629 .

[32] Afanasiev, P.; Bezverkhyy, I. Appl. Catal. A-GEN. 2007, 322, 129.

[33] Gaborit, V.; Allali, N.; Geantet, C.; Breysse, M.; Vrinat, M.; Danotl, M. Catal. Today 2000, 57, 267.

[34] Chai, Y. M.; An, G. J.; Liu, Y. Q.; Liu, C. G. Prog. Chem. 2007, 19, 234. (柴永明, 安高军, 柳云骐, 刘晨光, 化学进展, 2007, 19, 234.)

[35] Besenbacher, F.; Brorson, M.; Clausen, B. S.; Helveg, S.; Hinnemann, B.; Kibsgaard, J.; Lauritsen, J. V.; Moses, P. G.; Nørskovc, J. K.; Topsøe, H. Catal. Today 2008, 130, 86.

[36] Drescher, T.; Niefind, F.; Bensch, W.; Grünert, W. J. Am. Chem. Soc. 2012, $134,18896$.

[37] Prodhomme, P. Y.; Raybaud, P.; Toulhoat, H. J. Catal. 2011, 280, 178.

[38] Dinter, N.; Rusanen, M.; Raybaud, P.; Kasztelan, S.; Silva, P.; Toulhoat, H. J. Catal. 2010, 275, 117.

[39] Lauritsen, J. V.; Nyberg, M.; Nørskov, J. K.; Clausen, B. S.; Topsøe, H.; Lægsgaard, E.; Besenbacher, F. J. Catal. 2004, 224, 94.

[40] Wen, X. D.; Zeng, T.; Li, Y. W.; Wang, J.; Jiao, H. J. Phys. Chem. B 2005, 109, 18491.

[41] Lu, J. X. Chinese J. Struct. Chem. 1989, 5, 327. (卢嘉锡，结构化 学, 1989, 5, 327.)

[42] Huang, R. B.; Zhang, P.; Zhu, Y. B.; Zheng, L. S. Acta Phys-Chim. Sin. 1991，8，8. (黄荣涁, 张鹏, 朱永宝, 郑兰䔉, 物理化学学报, 1991, 8,8.)

[43] Popov, I.; Kunze, T.; Gemming, S.; Seifert, G. Eur. Phys. J. D. 2007, $45,439$.

[44] Popov, I.; Gemming, S.; Seifert, G. Phys. Rev. B 2007, 75, 245436.

[45] Seifert, G.; Tamuliene, J.; Gemming, S. Comput. Mater. Sci. 2006, 35,316 .

[46] Gemming, S.; Seifert, G. Appl. Phys. A 2006, 82, 175.

[47] Jiao, H. J.; Li, Y. W.; Delmon, B.; Halet, J. F. J. Am. Chem. Soc. 2001, 123, 7334.

[48] Bertram, N.; Kim, Y. D.; Ganteför, G.; Sun, Q.; Jena, P.; Tamliene, J.; Seifert, G. Chem. Phys. Lett. 2004, 396, 341.

[49] Liang, B.; Andrews, L. J. Phys. Chem. A 2002, 106, 3738.

[50] Liang, B.; Andrews, L. J. Phys. Chem. A 2002, 106, 6945.

[51] Yu, S. W.; Yin, L. Q.; Yao, L. F.; Li, M.; Xie, X. G. Chin. Chem. Lett. 2008, 19, 1008

[52] Yin, S.; Xie, Y.; Bernstein, E. R. J. Phys. Chem. A 2011, 115, 10266.

[53] Saha, A.; Raghavachari, K. J. Chem. Phys. 2013, 139, 204301.

[54] Saha, A.; Raghavachari, K. J. Chem. Phys. 2014, 141, 074305.

[55] Afanasiev, P.; Fischer, L.; Beauchesne, F.; Danot, M.; Gaborit, V.; Breysse, M. Catal. Lett. 2000, 64, 59

[56] Gaborit, V.; Allali, N.; Danot, M.; Geantet, C.; Cattenot, M.; Breysse, M.; Diehl, F. Catal. Today 2003, 78, 499.

[57] Aray, Y.; Zambrano, D.; Cornejo, M. H.; Ludeña, E. V.; Iza, P.; Vidal, A. B.; Coll, D. S.; Jimenez, D. M.; Henriquez, F.; Paredes, C. J. 
Phys. Chem. C 2014, 118, 27823.

[58] Ivanovskaya, V. V.; Heine, T.; Gemming, S.; Seifert, G. Phys. Status. Solidi. 2006, 243, 1757.

[59] Ivanovskaya, V. V.; Zobelli, A.; Gloter, A.; Brun, N.; Serin, V.; Colliex, C. Phys. Rev. B: Condens. Matter 2008, 78, 134104.

[60] Deepak, F. L.; Cohen, H.; Cohen, S.; Feldman, Y.; Popovitz-Biro, R.; Azulay, D.; Millo, O.; Tenne, R. J. Am. Chem. Soc. 2007, 129, 12549.

[61] Frisch, M. J.; Trucks, G. W.; Schlegel, H. B.; Scuseria, G. E.; Robb, M. A.; Cheeseman, J. R.; Montgomery, J. A., Jr.; Vreven, T.; Kudin, K. N.; Burant, J. C.; Millam, J. M.; Iyengar, S. S.; Tomasi, J.; Barone, V.; Mennucci, B.; Cossi, M.; Scalmani, G.; Rega, N.; Petersson, G. A.; Nakatsuji, H.; Hada, M.; Ehara, M.; Toyota, K.; Fukuda, R.; Hasegawa, J.; Ishida, M.; Nakajima, T.; Honda, Y.; Kitao, O.; Nakai, H.; Klene, M.; Li, X.; Knox, J. E.; Hratchian, H. P.; Cross, J. B.; Bakken, V.; Adamo, C.; Jaramillo, J.; Gomperts, R.; Stratmann, R. E.; Yazyev, O.; Austin, A. J.; Cammi, R.; Pomelli, C.; Ochterski, J. W.; Ayala, P. Y.; Morokuma, K.; Voth, G. A.; Salvador, P.; Dannenberg, J. J.; Zakrzewski, V. G.; Dapprich, S.; Daniels, A. D.; Strain, M. C.; Farkas, O.; Malick, D. K.; Rabuck, A. D.; Raghavachari, K.; Foresman, J. B.; Ortiz, J. V.; Cui, Q.; Baboul, A. G.; Clifford, S.; Cioslowski, J.; Stefanov, B. B.; Liu, G.; Liashenko, A.; Piskorz, P.; Komaromi, I.; Martin, R. L.; Fox, D. J.; Keith, T.; Al-Laham, M. A.; Peng, C. Y.; Nanayakkara, A.; Challacombe, M.; Gill, P. M. W.; Johnson, B.; Chen, W.; Wong, M. W.; Gonzalez, C.; Pople, J. A., Gaussian 03, Revision D. 01, Gaussian, Inc.: Wallingford, CT, 2004.

[62] Becke, A. D. J. Chem. Phys. 1993, 98, 1372.

[63] Lee, C.; Yang, W. T.; Parr, R. G. Phys. Rev. B 1988, 37, 785.

[64] Stephens, P. J.; Devlin, F. J.; Chabalowski, C. F.; Frisch, M. J. Phys. Chem. 1994, 98, 11623 .

[65] Schafer, A.; Huber, C.; Ahlrichs, R. J. Chem. Phys. 1994, 100, 5829

[66] Weigend, F.; Ahlrichs, R. Phys. Chem. Chem. Phys. 2005, 7, 3297.

[67] Eichkorn, K.; Weigend, F.; Treutler, O.; Ahlrichs, R. Theor. Chem. Acc. 1997, 97, 119. The exponents (included those of the polarization functions) and contraction coefficients can be retrieved from the following web-site: https: //bse. pnl. gov/bse/portal.

[68] Andrae, D.; Häußermann, U.; Dolg, M.; Stoll, H.; Preuß, H. Theor. Chim. Acta 1990, 77, 123.

[69] Kuchle, W.; Dolg, M.; Stoll, H.; Preuss, H. Pseudopotentials of the Stuttgart/Dresden Group 1998, revision August 11, 1998; <http: //www. theochem. uni-stuttgart. de/pseudopotentiale $>$.
[70] Dunning, T. H. Jr. J. Chem. Phys. 1989, 90, 1007.

[71] Martin, J. M. L.; Sundermann, A. J. Chem. Phys. 2001, 114, 3408.

[72] Woon, D. E.; Dunning, T. H. Jr. J. Chem. Phys. 1993, 98, 1358

[73] Dunning, T. H. Jr.; Peterson, K. A.; Wilson, A. K. J. Chem. Phys. 2001, 114, 9244.

[74] Purvis, G. D.; Bartlett, R. J. J. Chem. Phys. 1982, 76, 1910.

[75] Scuseria, G. E.; Janssen, C. L.; Schaefer III, H. F. J. Chem. Phys. 1988, 89, 7382 .

[76] Raghavachari, K.; Trucks, G. W.; Pople, J. A.; Head-Gordon, M. Chem. Phys. Lett. 1989, 157, 479.

[77] Watts, J. D.; Gauss, J.; Bartlett, R. J. J. Chem. Phys. 1993, 98, 8718.

[78] Bartlett, R. J.; Musial, M. Rev. Mod. Phys. 2007, 79, 291.

[79] Werner, H. J.; Knowles, P. J.; Knizia, G.; Manby, F. R.; Schütz, M.; Celani, P.; Györffy, W.; Kats, D.; Korona, T.; Lindh, R.; Mitrushenkov, A.; Rauhut, G.; Shamasundar, K. R.; Adler, T. B.; Amos, R. D.; Bernhardsson, A.; Berning, A.; Cooper, D. L.; Deegan, M. J. O.; Dobbyn, A. J.; Eckert, F.; Goll, E.; Hampel, C.; Hesselmann, A.; Hetzer, G.; Hrenar, T.; Jansen, G.; Köppl, C.; Liu, Y.; Lloyd, A. W.; Mata, R. A.; May, A. J.; McNicholas, S. J.; Meyer, W.; Mura, M. E.; Nicklaß, A.; O'Neill, D. P.; Palmieri, P.; Peng, D.; Pflüger, K.; Pitzer, R.; Reiher, M.; Shiozaki, T.; Stoll, H.; Stone, A. J.; Tarroni, R.; Thorsteinsson, T.; Wang, M., MOLPRO, Version 2010. 1, a package of ab initio programs, <http: //www. molpro. net $>$.

[80] Dennington, R. II; Keith, T.; Millam, J. GaussView, Version 4. 1. 2., Semichem Inc., Shawnee Mission, 2007.

[81] Tozer, D. J.; Handy, N. C. J. Chem. Phys. 1998, 109, 10180.

[82] Zhang, S.; Luo, C. G.; Li, H. Y.; Lu, C.; Li, G. Q.; Lu, Z. W. Mater. Chem. Phys. 2015, 160, 227.

[83] Zhang, S.; Zhang, Y.; Lu, Z.; Shen, X.; Li, G.; Peng, F.; Bu, X. J. Mater. Sci. 2016, 51, 9440.

[84] Merki, D.; Fierro, S.; Vrubel, H.; Hu, X. Chem. Sci. 2011, 2, 1262.

[85] Duchet, J. C.; Van-Oers, E. M.; De-Beer, V. H. J.; Prins, R. J. Catal. 1983, 80, 386.

[86] Afanasiev, P.; Jobic, H.; Lorentz, C.; Leverd, P.; Mastubayashi, N.; Piccolo, L.; Vrinat, M. J. Phys. Chem. C 2009, 113, 4139.

[87] Afanasiev, P. J. Catal. 2010, 269, 269.

[88] Allali, N.; Leblanc, A.; Danot, M.; Geantet, C.; Vrinat, M.; Breysse, M. Catal. Today 1996, 27, 137.

[89] Christe, K. O.; Dixon, D. A.; Mclemore, D.; Wilson, W. W.; Sheehy, J. A.; Boatz, J. A. J. Fluorine Chem. 1999, 101, 151.

[90] Li, S.; Dixon, D. A. J. Phys. Chem. A 2006, 110, 6231.

(Cheng, B.; Fan, Y.) 